\title{
Interpreting coral reef monitoring data: A guide for improved management decisions
}

Authors: Jason Flower ${ }^{\mathrm{a}, \mathrm{b},{ }^{*},}$, Juan Carlos Ortiz ${ }^{\mathrm{b}}$, Iliana Chollett ${ }^{\mathrm{c}, \mathrm{a}, \mathrm{b}}$, Sabah Abdullah ${ }^{\mathrm{b}}$, Carolina CastroSanguino ${ }^{\mathrm{b}}$, Karlo Hock ${ }^{\mathrm{b}}$, Vivian Lam ${ }^{\mathrm{b}}$, Peter J Mumby ${ }^{\mathrm{b}, \mathrm{a}}$

aMarine Spatial Ecology Lab, College of Life and Environmental Sciences, Geoffrey Pope Building, University of Exeter, Exeter EX4 4PS, UK

${ }^{b}$ Marine Spatial Ecology Lab, School of Biological Sciences, University of Queensland, St. Lucia Campus, Brisbane, QLD 4072, Australia

'Smithsonian Marine Station, Smithsonian Institution, Fort Pierce, FL 34949, USA

*Corresponding author. Current address: Sustainable Fisheries Group, Bren School of Environmental Science and Management, University of California, Santa Barbara, CA 93106, USA. Tel: +1805 4031691. E-mail address: jflowernet@aim.com

\section{Graphical abstract}

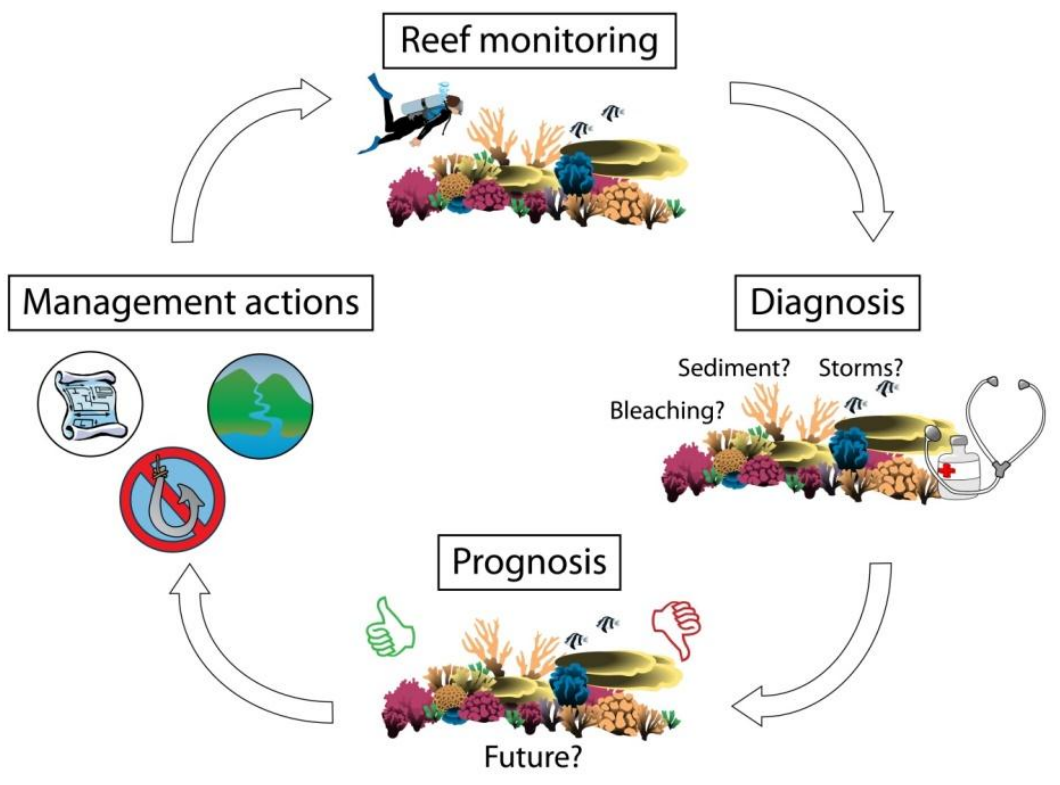

(C) 2016. This manuscript version is made available under the Elsevier user license http://www.elsevier.com/open-access/userlicense/1.0/ 


\begin{abstract}
Coral reef monitoring programmes exist in all regions of the world, recording reef attributes such as coral cover, fish biomass and macroalgal cover. Given the cost of such monitoring programs, and the degraded state of many of the world's reefs, understanding how reef monitoring data can be used to shape management decisions for coral reefs is a high priority. However, there is no general guide to understanding the ecological implications of the data in a format that can trigger a management response. We attempt to provide such a guide for interpreting the temporal trends in 41 coral reef monitoring attributes, recorded by seven of the largest reef monitoring programmes. We show that only a small subset of these attributes is required to identify the stressors that have impacted a reef (i.e. provide a diagnosis), as well as to estimate the likely recovery potential (prognosis). Two of the most useful indicators, turf algal canopy height and coral colony growth rate are not commonly measured, and we strongly recommend their inclusion in reef monitoring. The diagnosis and prognosis system that we have developed may help guide management actions and provides a foundation for further development as biological and ecological insights continue to grow.
\end{abstract}

Keywords: coral reef; monitoring; diagnostic; adaptive management; stressor; reef management

\title{
1 Introduction
}

Monitoring is a fundamental part of resource management, providing information on the state of the system which can be used to detect the impacts of natural and anthropogenic stressors, assess the potential recovery of the system, and measure the success of management interventions (Day, 2008; English et al., 1997; Legg and Nagy, 2006). Ecological monitoring of coral reefs, defined as repeated surveys collecting data on attributes such as abundance of fish and coral, has been conducted since reef survey techniques were first described in the 1970s (Jackson et al., 2014; Risk, 1999,1972 ). While all regions of the world have some form of reef monitoring, the regional comprehensiveness, level of replication, and depth of detail captured is highly variable globally (Wilkinson, 2008). With the increasing level of stress that reefs are being subjected to (Wolff et al., 2015), including the recent global bleaching event, it is more important than ever that reef monitoring data can be used to guide management action.

The dynamic nature of coral reefs and local differences in environmental conditions make interpreting changes in reef monitoring data difficult. For monitoring data to provide feedback to management, managers need a framework not only for collecting data but also for understanding and interpreting it (Houk and van Woesik, 2013; Renken and Mumby, 2009). Standard methods exist for surveying reefs (e.g. English et al., 1997; Hill and Wilkinson, 2004), with many programmes having developed regional variants, such as the Atlantic and Gulf Rapid Reef Assessment (AGRRA; Lang et al., 2010), the Caribbean Coastal Marine Productivity Program (CARICOMP, 2001), the Great Barrier Reef long-term monitoring program (Sweatman et al., 2008), and Reef Check (Hodgson et al., 2006). Survey methods have been subjected to extensive testing for accuracy and precision (Bohnsack and Bannerot, 1986; Brown et al., 2004; Carleton and Done, 1995; Leujak and Ormond, 2007; Ohlhorst et al., 1988), but the methods for interpreting the resulting data have not received so much attention. 
Much work has been done on biological assessments of ecosystem health and the identification and testing of biological indicators (bioindicators) in freshwater and estuarine ecosystems (Borja and Dauer, 2008; Karr and Chu, 1999). Development of bioindicators for coral reefs has lagged behind in part due to a lack of consistent, long-term datasets, differing sample methods, and the relative complexity of coral reef ecosystems (Jameson et al., 1998; McField and Kramer, 2006). Over the past decade there has been considerable work on developing and testing indicators for coral reefs (Bradley et al., 2008; Chabanet et al., 2005; Fisher et al., 2008; McField and Kramer, 2007) and a recent focus on indicators of resilience (McClanahan et al., 2012; Obura and Grimsditch, 2009). Some programmes at the regional level have developed indicators and thresholds that are used to interpret reef monitoring data and inform management. For example, the Healthy Reefs Initiative (HRI) for the Mesoamerican Barrier Reef was the first programme to develop specific target values for a variety of coral reef indicators (Kramer et al., 2015; McField and Kramer, 2007), several countries in the Eastern Caribbean have developed a reporting system modelled on the HRI indicators (CaribNode, 2016), and Bonaire, in the Southern Caribbean, has a monitoring program that uses a set of indicators to inform management (Steneck et al., 2016, 2005). However, there are still no general rules or guides for the interpretation of trends and patterns in reef monitoring data, to help answer questions such as: what are the likely causes of a given change in reef state? What sort of change should be considered potentially problematic?

To improve the use of coral reef monitoring data in management decisions a diagnostic approach has been suggested (Downs et al., 2005). The paradigm is similar to that used in medicine: a clinical examination of the subject (reef), which includes a review of the subject's history and an examination of the current state of health to identify the cause of the illness (Downs et al., 2005). So far, diagnostics of reef health have mainly employed indices of biotic integrity such as foraminiferan composition (Jameson et al., 2001, 1998) or used indicators at the sub-organism level, such as cellular changes (Downs et al., 2012, 2005; Hédouin and Berteaux-Lecellier, 2014). Since many of these indicators are not routinely collected by monitoring programmes, a diagnostic approach that focuses on making the best use of commonly collected, or easy to collect, reef monitoring data is needed for better integration of monitoring data with management actions.

To help close the adaptive management loop between monitoring data collection and management action, we first provide a guide to interpreting trends in reef attributes collected by major coral reef monitoring programmes. We then build on this information, selecting key indicators and combining their interpretation to provide a method for identifying the most likely stressors on the reef (i.e. a diagnosis). Taking the clinical approach one step further, we integrate the diagnostic results with indicators linked to reef recovery processes to provide a relative prognosis of reef health, which might help a reef manager target interventions. By reviewing reef attributes that are needed for diagnosis and prognosis, we were able to identify a minimum set of attributes to guide targeted management action, which might help increase the cost-effectiveness of reef monitoring worldwide.

\section{Methods}

\subsection{Interpreting changes in reef monitoring data}

A list of the reef attributes that are used for coral reef assessment was collated from the seven internationally recognised coral reef assessment programmes with published protocols. Four of the 
assessment programmes included are monitoring programmes and three (AGRRA, CARICOMP and Reef (heck) are reef assessment programmes that are often used for monitoring purposes.

For each attribute we provide an interpretation of its trend assuming that an observed trend would exceed two years to guard against - although not necessarily eradicate - measurement error or stochastic variability. We focus on trends that will negatively impact reef health, such as a decrease in coral cover or an increase in macroalgal cover. For coral, dead coral, macroalgal and turf algal cover, we included interpretation of both acute and chronic trends because they involve distinctly different ecological interpretations. We use a threshold of a $10 \%$ change in cover to define an acute change as smaller changes may constitute normal inter-annual variation (Graham et al., 2011).

For each trend in an attribute we considered: (1) main possible drivers of the trend; (2) other attributes to cross-reference to help confirm drivers of the trend; (3) the impact of the trend on reef ecological processes. Cross-referencing other attribute trends can narrow down the list of potential drivers and therefore help determine where management efforts could be focused.

Our list of ecosystem processes is drawn from a conceptual model of ecological feedback processes (Figure 1). The model does not attempt to incorporate all components of a reef ecosystem, but includes processes that are fundamental to the balance between a coral or algal-dominated reef, namely those that effect coral recruitment, growth and mortality (Hughes and Tanner, 2000).

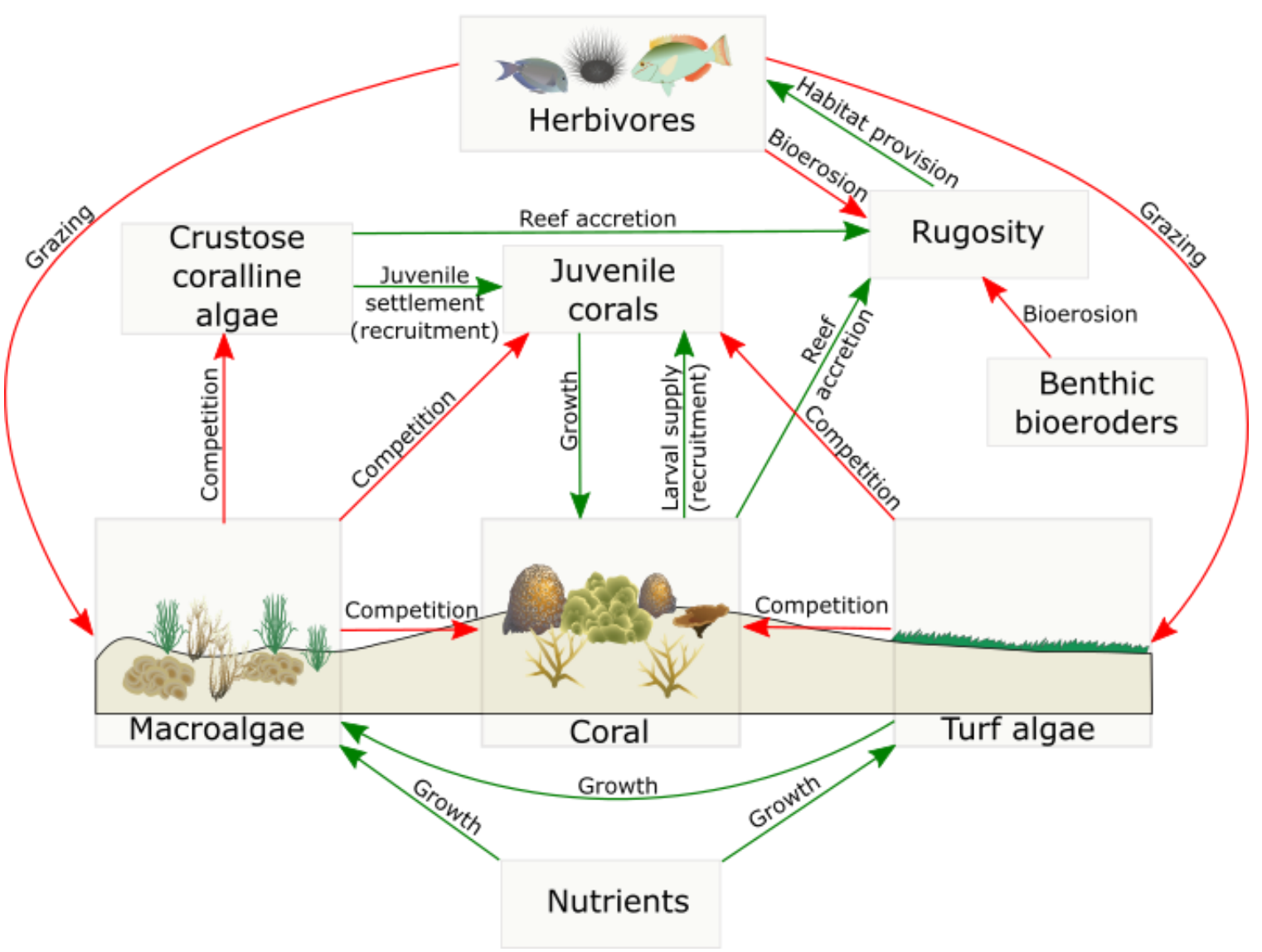

Figure 1. Main reef ecological feedback processes. Boxes show reef attributes, arrows show processes. Green arrows indicate a positive effect on the attribute; red arrows indicate a negative effect. Although not indicated, corals can also settle on short turf algae. 


\subsection{Diagnosing the main stressors affecting a reef}

We used an elimination approach to diagnose a stressor that has impacted or is impacting a reef. The approach uses a series of closed Yes/ No questions, involving knowledge of trends of indicators, to reach a diagnosis. The indicators were selected from the list of coral reef monitoring attributes (Table A1 Appendix A), local knowledge or information from open access internet databases. We selected indicators that are most strongly associated with a particular stressor or that could split groups of stressors (Table 1). For example, a recent thermal anomaly is a strong indicator of coral bleaching (a stressor). The indicators were arranged hierarchically into a decision tree, with each level either confirming or rejecting diagnoses. Decision trees have been used extensively in management guides, with several examples within the context of coral reefs (Edwards and Gomez, 2007; Marshall and Schuttenberg, 2006). They provide a simple way of presenting choices in a structured manner, without overwhelming the user with information.

Table 1. Indicators for diagnosis of stressors affecting a reef. Thresholds identified are general guidelines to indicate magnitude of change required for a 'Yes' response to the indicator.

\begin{tabular}{|c|c|c|c|}
\hline Indicator & $\begin{array}{l}\text { Diagnostic use } \\
\text { (indicator of) }\end{array}$ & $\begin{array}{l}\text { Thresholds and information sources (if not reef } \\
\text { monitoring data) }\end{array}$ & $\begin{array}{l}\text { Supporting } \\
\text { references }\end{array}$ \\
\hline $\begin{array}{l}\text { Sudden } \\
\text { decrease in } \\
\text { coral cover? }\end{array}$ & Acute stressor & $\begin{array}{l}\geq 10 \% \text { coral cover loss between consecutive data } \\
\text { points }\end{array}$ & $\begin{array}{l}\text { Graham et } \\
\text { al., } 2011\end{array}$ \\
\hline $\begin{array}{l}\text { Sudden } \\
\text { decrease in } \\
\text { rugosity? }\end{array}$ & $\begin{array}{l}\text { Physical damage } \\
\text { to reef }\end{array}$ & $\begin{array}{l}\geq 8 \% \text { decrease in rugosity between consecutive } \\
\text { data points }\end{array}$ & $\begin{array}{l}\text { Alvarez-Filip } \\
\text { et al., } 2011\end{array}$ \\
\hline Recent storm? & Storm & $\begin{array}{l}\text { Information from either local knowledge or } \\
\text { online via: } \\
\text { http://www.wmo.int/pages/prog/www/tcp/Advi } \\
\text { sories-RSMCs.html }\end{array}$ & $\mathrm{N} / \mathrm{A}$ \\
\hline $\begin{array}{l}\text { Recent } \\
\text { thermal } \\
\text { anomaly? }\end{array}$ & Coral bleaching & $\begin{array}{l}\text { Information from local observations or NOAAs } \\
\text { Coral Reef Watch programme: } \\
\text { http://coralreefwatch.noaa.gov/ }\end{array}$ & $\begin{array}{l}\text { Liu et al., } \\
2014\end{array}$ \\
\hline $\begin{array}{l}\text { Crown-of- } \\
\text { thorns starfish } \\
\text { outbreak? }{ }^{*}\end{array}$ & $\begin{array}{l}\text { Crown-of-thorns } \\
\text { starfish } \\
\text { outbreak }\end{array}$ & $\begin{array}{l}\text { Threshold density for outbreak } \geq 1500 \text { adult } \\
\text { starfish } \mathrm{km}^{-2}\end{array}$ & $\begin{array}{l}\text { Moran and } \\
\text { De'ath, } \\
1992\end{array}$ \\
\hline $\begin{array}{l}\text { Species } \\
\text { specific coral } \\
\text { loss? }\end{array}$ & $\begin{array}{l}\text { Disease } \\
\text { outbreak }\end{array}$ & $\begin{array}{l}\geq 10 \% \text { coral cover loss (Graham et al., 2011) of } \\
\text { coral species particularly affected by coral } \\
\text { diseases }\end{array}$ & $\begin{array}{l}\text { Raymundo } \\
\text { et al., 2008, } \\
\text { appendix } 5\end{array}$ \\
\hline
\end{tabular}




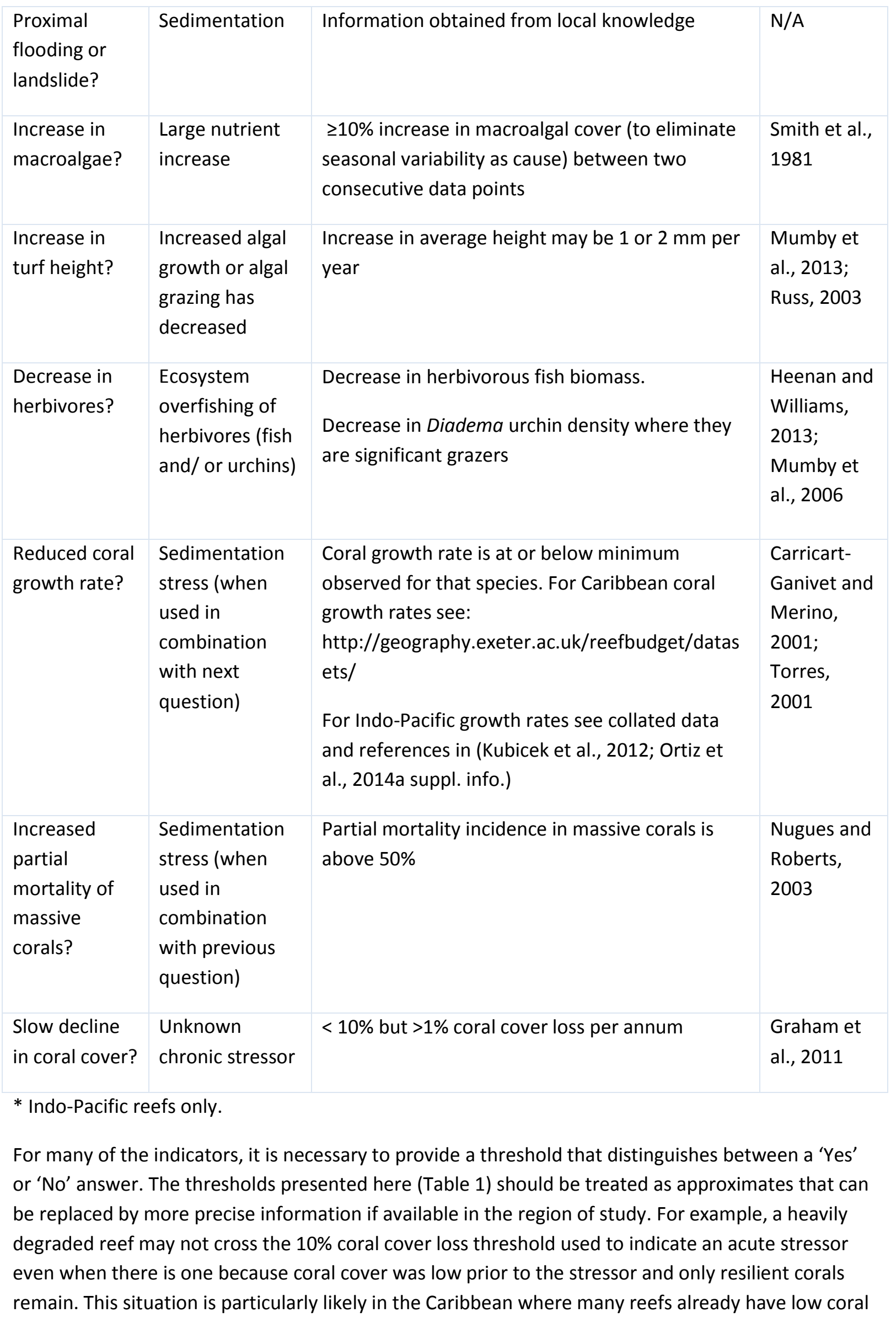


cover, high macroalgal biomass and low herbivore biomass (Jackson et al., 2014). In these cases, lower threshold values may have to be used, which could be approximated by using data on the impact of stressors on comparable reefs within the region.

The chronic stressors of climate change (i.e. ocean acidification and the effects of increasing water temperatures on coral growth rates and fecundity) are not included as diagnosable stressors, as they are problematic to detect using common reef monitoring data and are also difficult to disentangle from other stressors (Mumby and van Woesik, 2014).

\subsection{Relative prognosis of reef health}

\subsubsection{Selection of indicators}

To assess the prognosis for a reef, we first had to identify a set of indicators that provide the most information on the potential of the reef to recover. Since the process of coral recovery is fundamentally driven by recruitment, growth and mortality of corals, we focused on identifying suitable indicators of change in these processes. Indicators were identified using the same conceptual model of a coral reef used for diagnosis (Figure 1). Crustose coralline algae (CCA) were excluded at this point because of their rarity in much of the Caribbean (Newman et al., 2006 suppl.), but they could be added for Indo-Pacific analyses. The indicators identified as relevant to assess the prognosis for a reef were turf, macroalgae, herbivores, juvenile corals and coral growth. Where multiple metrics are available for measuring an indicator, we used that which provided the clearest signal of the ability of the reef to recover (Table 2). For cases where the stressor to the reef has already been diagnosed as sedimentation, a coral disease outbreak or a crown-of-thorns starfish outbreak, there are specific prognosis indicators that help determine how severe the continuing impact on the reef will be (Table 2).

Table 2. Prognosis indicators, their scores and reasons for inclusion in prognosis. Addition scores results in a 'prognosis score' for a reef, with the highest value representing the worst possible prognosis.

\begin{tabular}{|c|c|c|c|}
\hline Indicator & $\begin{array}{l}\text { Conditions for inclusion in } \\
\text { prognosis }\end{array}$ & $\begin{array}{l}\text { Score for } \\
\text { Yes answer } \\
(\mathrm{No}=0)\end{array}$ & $\begin{array}{l}\text { Justification for inclusion } \\
\text { in prognosis and scoring }\end{array}$ \\
\hline $\begin{array}{l}\text { Turf canopy height } \\
\text { increasing? }\end{array}$ & None & 1 & $\begin{array}{l}\text { Coral recruitment } \\
\text { decreases with increases } \\
\text { in turf canopy height } \\
\text { (Arnold et al., 2010; Birrell } \\
\text { et al., 2008; Vermeij et al., } \\
\text { 2010) }\end{array}$ \\
\hline $\begin{array}{l}\text { Juvenile coral density } \\
\text { decreasing? }\end{array}$ & None & 1 & $\begin{array}{l}\text { Indicates reduced } \\
\text { recruitment - recovery of } \\
\text { reef impaired (Hughes and } \\
\text { Tanner, 2000) and } \\
\text { possible reduction of }\end{array}$ \\
\hline
\end{tabular}




\begin{tabular}{|c|c|c|c|}
\hline & & & $\begin{array}{l}\text { genetic diversity } \\
\text { (Knowlton, 2001) }\end{array}$ \\
\hline $\begin{array}{l}\text { Coral community } \\
\text { dominated by sediment } \\
\text { sensitive corals? } \\
\text { (for heavy sedimentation, e.g. } \\
\text { due to beach nourishment or } \\
\text { proximate construction, } \\
\text { always answer "Yes" } \\
\text { regardless of coral } \\
\text { community) }\end{array}$ & $\begin{array}{l}\text { only for stressor = } \\
\text { sedimentation }\end{array}$ & 1 & $\begin{array}{l}\text { Recovery will be impaired } \\
\text { due to coral mortality, } \\
\text { slow growth and reduced } \\
\text { fecundity and recruitment } \\
\text { (Fabricius, 2005; Rogers, } \\
\text { 1990) }\end{array}$ \\
\hline $\begin{array}{l}\text { Coral disease incidence } \\
\text { increasing? }\end{array}$ & only for stressor = disease & 1 & $\begin{array}{l}\text { Recovery will be impaired } \\
\text { due to coral mortality, } \\
\text { slow growth and reduced } \\
\text { fecundity (Weil and } \\
\text { Rogers, 2011) }\end{array}$ \\
\hline $\begin{array}{l}\text { COTS density higher than } \\
\text { threshold? }\end{array}$ & $\begin{array}{l}\text { only for stressor = COTS } \\
\text { outbreak } \\
\text { Threshold density for COTS } \\
\text { outbreak } \geq 1500 \text { COTS km² } \\
\text { (Moran and De'ath, 1992). }\end{array}$ & 1 & $\begin{array}{l}\text { Coral mortality exceeds } \\
\text { rate of recovery (Moran } \\
\text { and De'ath, 1992; } \\
\text { Pratchett et al., 2014) }\end{array}$ \\
\hline \multirow[t]{2}{*}{$\begin{array}{l}\text { Coral community } \\
\text { dominated by slow } \\
\text { growing corals? }\end{array}$} & Acute stressor diagnosed? & 2 & $\begin{array}{l}\text { Recovery impaired as } \\
\text { corals will tend to be } \\
\text { outcompeted for space by } \\
\text { macroalgae (Mumby et } \\
\text { al., 2007; Ortiz et al., } \\
\text { 2014b) }\end{array}$ \\
\hline & $\begin{array}{l}\text { Chronic stressor } \\
\text { diagnosed? }\end{array}$ & 1 & $\begin{array}{l}\text { As above, but less severe } \\
\text { as reduction in coral cover } \\
\text { is less than in acute } \\
\text { stressor case }\end{array}$ \\
\hline $\begin{array}{l}\text { Macroalgal cover } \\
\text { increasing? }\end{array}$ & $\begin{array}{l}\text { If community dominated } \\
\text { by slow growing corals }\end{array}$ & 2 & $\begin{array}{l}\text { Corals will tend to be } \\
\text { outcompeted for space by } \\
\text { macroalgae (Nugues and } \\
\text { Bak, 2006; Rasher and } \\
\text { Hay, 2010). Macroalgae } \\
\text { reduce space available for } \\
\text { recruitment and increase } \\
\text { mortality of juvenile corals } \\
\text { (Box and Mumby, 2007). }\end{array}$ \\
\hline
\end{tabular}




\begin{tabular}{|c|c|c|c|}
\hline & If not & 1 & $\begin{array}{l}\text { As above, but faster } \\
\text { growing corals are more } \\
\text { competitive for space. }\end{array}$ \\
\hline \multirow[t]{2}{*}{$\begin{array}{l}\text { Decrease in herbivores? } \\
\text { (Decrease in herbivorous } \\
\text { fish biomass and/ or } \\
\text { Diadema urchin density } \\
\text { where they are significant } \\
\text { grazers) }\end{array}$} & $\begin{array}{l}\text { If community dominated } \\
\text { by slow growing corals }\end{array}$ & 2 & $\begin{array}{l}\text { Herbivory is important for } \\
\text { control of algae (Burkepile } \\
\text { and Hay, 2009; Williams } \\
\text { and Polunin, 2001). } \\
\text { Slower growing corals will } \\
\text { struggle to outcompete } \\
\text { algae for space (Ortiz et } \\
\text { al., 2014a; Roff and } \\
\text { Mumby, 2012). }\end{array}$ \\
\hline & If not & 1 & $\begin{array}{l}\text { As above, but faster } \\
\text { growing corals are more } \\
\text { competitive for space }\end{array}$ \\
\hline \multirow[t]{2}{*}{$\begin{array}{l}\text { Coral growth rate } \\
\text { decreasing? }\end{array}$} & $\begin{array}{l}\text { If community dominated } \\
\text { by slow growing corals }\end{array}$ & 2 & $\begin{array}{l}\text { Slower growing corals will } \\
\text { struggle to outcompete } \\
\text { algae for space (Ortiz et } \\
\text { al., 2014a; Roff and } \\
\text { Mumby, 2012). }\end{array}$ \\
\hline & If not & 1 & $\begin{array}{l}\text { As above, but faster } \\
\text { growing corals are more } \\
\text { competitive for space }\end{array}$ \\
\hline
\end{tabular}

\subsubsection{Scoring system}

To place a reef on a relative prognosis scale from 'best potential future outcome' to 'worst potential future outcome' we provided a score for each indicator based on evidence from the published literature. As with the diagnosis, the indicators are assessed via closed questions with simple 'Yes' or 'No' answers. A score of either 1 or 2 is given for a 'Yes' answer to an indicator question, reflecting a negative impact on reef recovery. A score of 2 is given where there is strong evidence from the literature that two indicators will interact synergistically, with the emergent outcome of their combined impact likely to be worse than the simple addition of the two individual effects (Table 2). Working through each indicator for which the user has data available and summing the scores obtained provides a prognosis score, with the lowest score indicating the best prognosis. The more indicators that the user has data for, the broader the spectrum of possible prognoses; for example, with data for only turf canopy height, only scores of 0 or 1 are possible, but if data for coral growth rate are also available, scores from 0 to 3 are possible. Using more indicators will also give the user more assurance that the prognosis is likely to be correct. To reduce redundancy, indicators that have already been used in the diagnosis should not be used to calculate the prognosis, as this would simply increase all potential prognosis scores by 0,1 or 2 (depending on the indicator) rather than increase the difference between the prognoses. For example, if the chronic stressor 'nutrient 
increase' is diagnosed for a reef, the user must have used the indicators turf height and herbivore biomass to get to this diagnosis. Using these indicators again for the prognosis does not provide new information, it only adds a constant to all the possible prognosis scores.

\section{Results and Discussion}

\subsection{Interpreting changes in reef monitoring data}

A total of 41 reef attributes were collated from the monitoring programmes assessed. We grouped the attributes into four categories: Scleractinian coral, algae, other benthic attributes and fish (Table A1 in Appendix A). The benthic cover attributes of coral, macroalgae, sponge, sediment, bare substrate and other sessile organisms were included in all programmes. Coral size distribution, coral growth and sponge height and abundance were each included in only one programme. One monitoring programme (Coral Reef Evaluation and Monitoring Project, South Florida) does not include fish in their monitoring, and of the remaining six, two (Reef Check and AIMS LTMP) only include abundance and length for a small number of fish indicator species.

For each reef monitoring attribute, there are often multiple possible drivers of a trend which are not mutually exclusive; for example, a decrease in coral juvenile density could be driven by a decrease in substrate suitable for recruitment, an acute stressor causing juvenile mortality, a decrease in coral larval supply, and/ or decreased water quality (Table 3 column 3 ). By cross-referencing those attributes suggested in Table 3 column 4, a user should be able to confirm which is the most likely driver of a trend. 
Table 3. Interpretation of coral reef monitoring attributes.

\begin{tabular}{|c|c|c|c|c|c|}
\hline Attribute & $\begin{array}{l}\text { What is the } \\
\text { trend? }\end{array}$ & $\begin{array}{l}\text { Main possible drivers of } \\
\text { trend }\end{array}$ & $\begin{array}{l}\text { Other attributes to } \\
\text { cross reference to } \\
\text { confirm driver }\end{array}$ & Supporting references & $\begin{array}{l}\text { Direct impact of } \\
\text { trend on ecological } \\
\text { processes }\end{array}$ \\
\hline
\end{tabular}

\section{Scleractinian coral:}

Coral juvenile density Decrease

\section{Decrease in substrate Macroalgal cover, turf suitable for recruitment, e.g. due to thick algal turfs, fleshy macroalgae or cyanobacteria \\ canopy height, \\ cyanobacteria cover, \\ herbivorous fish \\ biomass, Diadema \\ density}

\section{Acute stressor ${ }^{\mathrm{a}}$ caused juvenile mortality}

Decrease in coral larval supply

Decreased water quality (including sedimentation)
Coral cover, rugosity, coral bleaching

prevalence/ incidence

Coral cover by genus/ species, coral cover of nearby reefs

Water quality data, sediment trap data
Box and Mumby, 2007;

Kuffner et al., 2006

Mumby, 1999

Gilmour et al., 2013;

Jones et al., 2009;

Vermeij and Sandin,

2008

Cortés and Risk, 1985;

Ritson-Williams et al.,

2009 


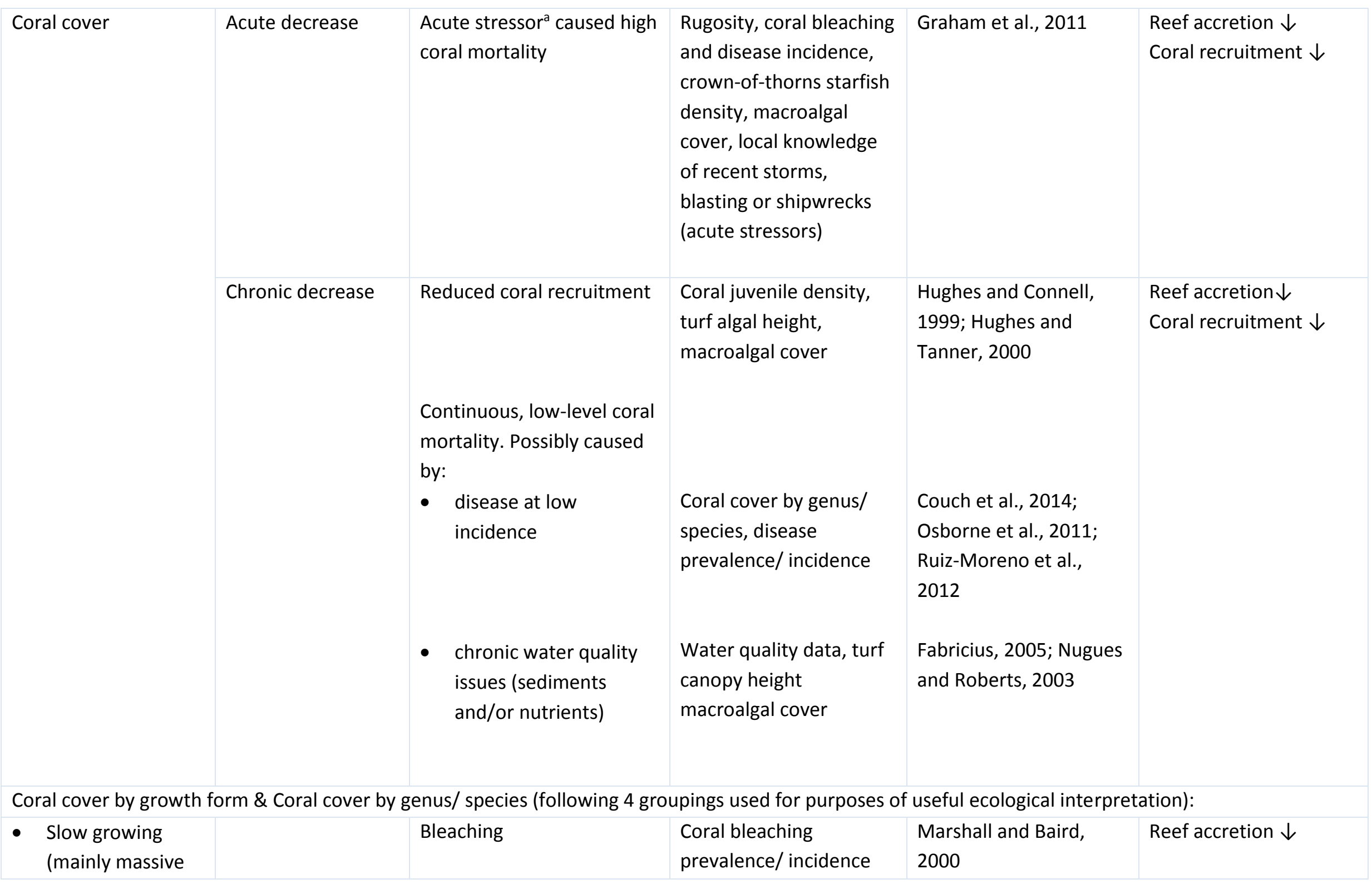


corals)

\begin{tabular}{|c|c|c|c|c|}
\hline \multirow[t]{4}{*}{$\begin{array}{l}\text { Decrease in relative } \\
\text { abundance } \\
\text { (for reefs with high } \\
\text { relative abundance } \\
\quad \text { of group) }\end{array}$} & Sedimentation & $\begin{array}{l}\text { Coral disease } \\
\text { prevalence/ incidence }\end{array}$ & $\begin{array}{l}\text { Raymundo et al., 2008; } \\
\text { Ruiz-Moreno et al., } \\
2012 \\
\text { Fabricius, 2005; Nugues } \\
\text { and Roberts, } 2003\end{array}$ & \\
\hline & $\begin{array}{l}\text { Bleaching } \\
\text { Disease (for acroporids in } \\
\text { Atlantic) } \\
\text { Storm }\end{array}$ & $\begin{array}{l}\text { Coral bleaching } \\
\text { prevalence/ incidence } \\
\text { Coral disease } \\
\text { prevalence/ incidence } \\
\text { Rugosity }\end{array}$ & $\begin{array}{l}\text { McClanahan et al., } 2007 \\
\text { Aronson and Precht, } \\
2001 \\
\text { Madin, } 2005\end{array}$ & $\begin{array}{l}\text { Habitat provision } \downarrow \\
\text { Reef accretion } \downarrow\end{array}$ \\
\hline & Disease & $\begin{array}{l}\text { Coral disease } \\
\text { prevalence/ incidence }\end{array}$ & $\begin{array}{l}\text { Raymundo et al., 2008; } \\
\text { Ruiz-Moreno et al., } \\
2012\end{array}$ & Reef accretion $\downarrow$ \\
\hline & $\begin{array}{l}\text { Increased sediment on reef } \\
\text { e.g. due to deforestation of } \\
\text { surrounding land }\end{array}$ & $\begin{array}{l}\text { Sediment trap data, } \\
\text { coral partial mortality, } \\
\text { coral growth rate }\end{array}$ & $\begin{array}{l}\text { Fabricius, 2005; Nugues } \\
\text { and Roberts, } 2003\end{array}$ & Reef accretion $\downarrow$ \\
\hline $\begin{array}{l}\text { Decrease in } \\
\text { abundance of large } \\
\text { corals }\end{array}$ & $\begin{array}{l}\text { Coral colony mortality } \\
\text { (partial or whole) due to } \\
\text { stressor }\end{array}$ & $\begin{array}{l}\text { Coral cover, rugosity, } \\
\text { coral bleaching and } \\
\text { disease prevalence/ } \\
\text { incidence }\end{array}$ & $\begin{array}{l}\text { Alvarado-Chacón and } \\
\text { Acosta, 2009; Hughes } \\
\text { and Jackson, } 1980\end{array}$ & Reef accretion $\downarrow$ \\
\hline
\end{tabular}

- Fast growing

(mainly branching and plating

corals)

- Disease sensitive corals

- Sediment sensitive corals

Coral size distribution corals

\section{Coral disease}

prevalence/ incidence

Sediment trap data,

coral partial mortality,

Coral bleaching

prevalence/ incidence

Coral disease

prevalence/ incidence

Rugosity

Coral disease

prevalence/ incidence

Sediment trap data,

coral partial mortality

Coral cover, rugosity,

disease prevalence/

incidence
Raymundo et al., 2008

Ruiz-Moreno et al.,

and Roberts, 2003

McClanahan et al., 2007

Aronson and Precht,

2001

Madin, 2005

Raymundo et al., 2008; $\quad$ Reef accretion $\downarrow$

Ruiz-Moreno et al.,

and Roberts, 2003

Alvarado-Chacón and and Jackson, 1980 


\begin{tabular}{|c|c|c|c|c|c|}
\hline & & $\begin{array}{l}\text { If coral mortality only } \\
\text { partial, may result in } \\
\text { increase in abundance of } \\
\text { small corals }\end{array}$ & $\begin{array}{l}\text { Coral partial mortality, } \\
\text { abundance of small } \\
\text { corals (see below) }\end{array}$ & & \\
\hline & $\begin{array}{l}\text { Decrease in } \\
\text { abundance of small } \\
\text { corals }\end{array}$ & $\begin{array}{l}\text { Decrease in coral } \\
\text { recruitment }\end{array}$ & Coral juvenile density & $\begin{array}{l}\text { Alvarado-Chacón and } \\
\text { Acosta, 2009; Hughes } \\
\text { and Jackson, } 1980\end{array}$ & Reef accretion $\downarrow$ \\
\hline & $\begin{array}{l}\text { Increase in } \\
\text { abundance of small } \\
\text { corals }\end{array}$ & $\begin{array}{l}\text { Increased partial mortality } \\
\text { of large corals (fission of } \\
\text { colonies) } \\
\text { Increased coral } \\
\text { recruitment }\end{array}$ & $\begin{array}{l}\text { Coral partial mortality, } \\
\text { abundance of large } \\
\text { corals (see above) } \\
\text { Coral juvenile density }\end{array}$ & $\begin{array}{l}\text { Gilmour et al., 2013; } \\
\text { Hughes and Jackson, } \\
\text { 1980; Lewis, } 1997\end{array}$ & \\
\hline Coral growth & Decrease & $\begin{array}{l}\text { Increased sedimentation } \\
\text { on reef } \\
\text { Current or past presence of } \\
\text { disease or bleaching } \\
\text { Increased algal competition }\end{array}$ & $\begin{array}{l}\text { Sedimentation and/ or } \\
\text { water quality data, coral } \\
\text { cover by species } \\
\text { Disease/ bleaching } \\
\text { prevalence/ incidence, } \\
\text { coral cover by species } \\
\text { Coral cover, macroalgal } \\
\text { cover, turf cover, turf } \\
\text { canopy height }\end{array}$ & $\begin{array}{l}\text { Carricart-Ganivet and } \\
\text { Merino, 2001; Torres, } \\
2001 \\
\text { Gladfelter, 1982; } \\
\text { Goreau and Macfarlane, } \\
1990 \\
\text { Box and Mumby, 2007; } \\
\text { Tanner, } 1995\end{array}$ & Reef accretion $\downarrow$ \\
\hline
\end{tabular}




\begin{tabular}{|c|c|c|c|c|c|}
\hline $\begin{array}{l}\text { Coral predation } \\
\text { (abundance of } \\
\text { predators or tissue } \\
\text { scars) }\end{array}$ & Increase & $\begin{array}{l}\text { Increased abundance of } \\
\text { predators and/or coral } \\
\text { predation rates }\end{array}$ & $\begin{array}{l}\text { Coral cover by genus/ } \\
\text { species, abundance of } \\
\text { coral predators }\end{array}$ & Rotjan and Lewis, 2008 & $\begin{array}{l}\text { Reef accretion } \downarrow \\
\text { Bioerosion } \uparrow\end{array}$ \\
\hline Coral partial mortality & Increase & $\begin{array}{l}\text { Chronic stressor }{ }^{\mathrm{b}} \text { causing } \\
\text { partial coral mortality. } \\
\text { Most likely sources: } \\
\text { - Sediment } \\
\text { - Coral disease } \\
\text { - Coral bleaching } \\
\text { - Predation }\end{array}$ & $\begin{array}{l}\text { Coral growth rate, coral } \\
\text { size distribution, } \\
\text { sediment/ water quality } \\
\text { data } \\
\text { Coral disease } \\
\text { prevalence/ incidence } \\
\text { Coral bleaching } \\
\text { incidence } \\
\text { Coral predation }\end{array}$ & $\begin{array}{l}\text { Fabricius, 2005; Nugues } \\
\text { and Roberts, } 2003 \\
\text { Jones et al., } 2004 \\
\text { Baird and Marshall, } \\
2002 \\
\text { Garzón-Ferreira et al., } \\
2005\end{array}$ & $\begin{array}{l}\text { Reef accretion } \downarrow \\
\text { Coral colony growth } \downarrow\end{array}$ \\
\hline $\begin{array}{l}\text { Coral disease } \\
\text { prevalence }\end{array}$ & Increase & $\begin{array}{l}\text { Favourable environmental } \\
\text { conditions, possibly: } \\
\text { - extreme temperatures } \\
\text { (frequently disease } \\
\text { following coral } \\
\text { bleaching) }\end{array}$ & $\begin{array}{l}\text { Thermal anomaly data, } \\
\text { coral bleaching } \\
\text { prevalence/incidence }\end{array}$ & $\begin{array}{l}\text { Harvell et al., 2007; } \\
\text { Randall and van Woesik, } \\
\text { 2015; Ruiz-Moreno et } \\
\text { al., } 2012\end{array}$ & $\begin{array}{l}\text { Reef accretion } \downarrow \\
\text { Coral colony growth } \downarrow\end{array}$ \\
\hline
\end{tabular}




\begin{tabular}{|c|c|c|c|c|c|}
\hline & & - reduced water quality & $\begin{array}{l}\text { Sediment/ water quality } \\
\text { data, coral growth rate, } \\
\text { coral partial mortality }\end{array}$ & $\begin{array}{l}\text { Haapkylä et al., 2011; } \\
\text { Harvell et al., 2007; } \\
\text { Thurber et al., } 2013\end{array}$ & \\
\hline $\begin{array}{l}\text { Coral bleaching } \\
\text { prevalence }\end{array}$ & Increase & $\begin{array}{l}\text { Coral bleaching due to } \\
\text { elevated water } \\
\text { temperatures (may be } \\
\text { linked to weakening of } \\
\text { resilience by chronic } \\
\text { stressors such as increased } \\
\text { nutrient levels) } \\
\text { Other possible causal } \\
\text { stressors: } \\
\text { - decreased water } \\
\text { - temperature } \\
\text { - } \text { solar radiation } \\
\text { - } \text { bacterial infection }\end{array}$ & $\begin{array}{l}\text { Thermal anomaly data, } \\
\text { water quality data, coral } \\
\text { disease prevalence/ } \\
\text { incidence (may increase } \\
\text { following bleaching) }\end{array}$ & $\begin{array}{l}\text { Brown, 1997; Eakin et } \\
\text { al., 2010; Hoegh- } \\
\text { Guldberg, 1999; Lesser, } \\
2011\end{array}$ & $\begin{array}{l}\text { Reef accretion } \downarrow \\
\text { Coral colony growth } \downarrow\end{array}$ \\
\hline \multicolumn{6}{|l|}{ Algae: } \\
\hline $\begin{array}{l}\text { Macroalgal cover } \\
\text { (fleshy macroalgae) }\end{array}$ & Acute increase & $\begin{array}{l}\text { Acute stressor resulting in } \\
\text { increase in substrate } \\
\text { available for macroalgal } \\
\text { colonization due to coral } \\
\text { mortality }\end{array}$ & $\begin{array}{l}\text { Coral cover, rugosity, } \\
\text { coral bleaching and } \\
\text { disease incidence, } \\
\text { crown-of-thorns starfish } \\
\text { density, local knowledge } \\
\text { of recent storms, } \\
\text { blasting or shipwrecks }\end{array}$ & $\begin{array}{l}\text { Diaz-Pulido et al., 2009; } \\
\text { Hatcher, 1984; Mumby } \\
\text { et al., } 2005\end{array}$ & $\begin{array}{l}\text { Coral-algal } \\
\text { competition } \uparrow \\
\text { Coral growth } \downarrow \\
\text { Coral recruitment } \downarrow\end{array}$ \\
\hline
\end{tabular}




\begin{tabular}{|c|c|c|c|c|c|}
\hline & & & (acute stressors) & & \\
\hline & & Large increase in nutrients & Water quality data & Smith et al., 1981 & \\
\hline & Chronic Increase & $\begin{array}{l}\text { Decrease in herbivores } \\
\text { Increase in nutrients }\end{array}$ & $\begin{array}{l}\text { Herbivore biomass, } \\
\text { fisheries data } \\
\text { Nutrient levels }\end{array}$ & $\begin{array}{l}\text { Williams and Polunin, } \\
2001 \\
\text { Burkepile and Hay, } \\
\text { 2006; Cooper et al., } \\
2009\end{array}$ & \\
\hline $\begin{array}{l}\text { Macroalgal canopy } \\
\text { height }\end{array}$ & Increase & $\begin{array}{l}\text { Decrease in herbivores } \\
\text { Increase in nutrients }\end{array}$ & $\begin{array}{l}\text { Herbivore biomass, } \\
\text { fisheries data } \\
\text { Nutrient levels }\end{array}$ & $\begin{array}{l}\text { Williams and Polunin, } \\
2001 \\
\text { Burkepile and Hay, } \\
\text { 2006; Cooper et al., } \\
2009\end{array}$ & $\begin{array}{l}\text { Coral-algal } \\
\text { competition } \uparrow \\
\text { Coral growth } \downarrow \\
\text { Coral recruitment } \downarrow\end{array}$ \\
\hline Macroalgal cover b) & cies/genus (see $n$ & algal functional groups - fle & y macroalgae, calcareous & acroalgae, CCA and turf) & \\
\hline Turf cover & Acute Increase & $\begin{array}{l}\text { Acute stressor }{ }^{a} \text { resulting in } \\
\text { increase in substrate } \\
\text { available for turf algal } \\
\text { colonization due to coral } \\
\text { mortality }\end{array}$ & $\begin{array}{l}\text { Coral cover, rugosity, } \\
\text { coral bleaching and } \\
\text { disease incidence, } \\
\text { crown-of-thorns starfish } \\
\text { density, local knowledge } \\
\text { of recent storms, } \\
\text { blasting or shipwrecks } \\
\text { (acute stressors) }\end{array}$ & $\begin{array}{l}\text { Diaz-Pulido and } \\
\text { McCook, 2002; Williams } \\
\text { et al., } 2001\end{array}$ & $\begin{array}{l}\text { Coral-algal } \\
\text { competition } \uparrow \\
\text { Coral recruitment } \downarrow\end{array}$ \\
\hline
\end{tabular}




\begin{tabular}{|c|c|c|c|c|c|}
\hline & & $\begin{array}{l}\text { Large increase in nutrients } \\
\text { due to increased terrestrial } \\
\text { run-off, e.g. from storms }\end{array}$ & Water quality data & Russ and McCook, 1999 & \\
\hline & Chronic Increase & $\begin{array}{l}\text { Decrease in herbivores } \\
\text { Increase in nutrients }\end{array}$ & $\begin{array}{l}\text { Herbivores biomass, } \\
\text { fisheries data } \\
\text { Water quality data }\end{array}$ & $\begin{array}{l}\text { Arnold et al., 2010; } \\
\text { Mumby et al., } 2013 \\
\text { McClanahan et al., } \\
2002 \text {; Vermeij et al., } \\
2010\end{array}$ & $\begin{array}{l}\text { Coral-algal } \\
\text { competition } \uparrow \\
\text { Coral recruitment } \downarrow\end{array}$ \\
\hline Turf canopy height & Increase & $\begin{array}{l}\text { Decrease in herbivores } \\
\text { Increase in nutrients } \\
\text { Increase in dead coral } \\
\text { (substrate available for } \\
\text { grazing) }\end{array}$ & $\begin{array}{l}\text { Herbivore biomass, } \\
\text { fisheries data } \\
\text { Water quality data }\end{array}$ & $\begin{array}{l}\text { Arnold et al., 2010; } \\
\text { Mumby et al., 2013; } \\
\text { Vermeij et al., } 2010 \\
\text { McClanahan et al., } \\
2002 \text {; Vermeij et al., } \\
2010 \\
\text { Diaz-Pulido and } \\
\text { McCook, 2002; Williams } \\
\text { et al., } 2001\end{array}$ & Coral recruitment $\downarrow$ \\
\hline $\begin{array}{l}\text { Crustose coralline } \\
\text { algae }(C C A) \text { cover }\end{array}$ & Decrease & $\begin{array}{l}\text { Increased sediment or } \\
\text { pollutants }\end{array}$ & $\begin{array}{l}\text { Sediment trap data, } \\
\text { coral partial mortality, } \\
\text { coral growth rate }\end{array}$ & $\begin{array}{l}\text { Fabricius and De'ath, } \\
\text { 2001; Harrington et al., } \\
\text { 2005; Steneck, } 1997\end{array}$ & $\begin{array}{l}\text { Coral recruitment } \downarrow \\
\text { Reef accretion } \downarrow\end{array}$ \\
\hline
\end{tabular}




\begin{tabular}{|c|c|c|c|c|c|}
\hline & & $\begin{array}{l}\text { Reduction in herbivores } \\
\text { CCA disease, possibly } \\
\text { linked to increased water } \\
\text { temperature }\end{array}$ & $\begin{array}{l}\text { Herbivore biomass } \\
\text { CCA disease incidence, } \\
\text { water quality, sea } \\
\text { surface temperature } \\
\text { data }\end{array}$ & $\begin{array}{l}\text { Burkepile and Hay, } \\
\text { 2009, } 2006 \\
\text { Miller et al., 2013; } \\
\text { Quéré et al., } 2015\end{array}$ & \\
\hline Calcareous algal cover & Increase & Reduction in herbivores & Herbivore biomass & Ferrari et al., 2012 & $\begin{array}{l}\text { Coral-algal competition } \\
\uparrow\end{array}$ \\
\hline Cyanobacteria cover & Increase & $\begin{array}{l}\text { Increased terrestrial run- } \\
\text { off, particularly nutrients }\end{array}$ & Water quality data & $\begin{array}{l}\text { Albert et al., 2005; } \\
\text { Brocke et al., 2015; } \\
\text { Kuffner et al., } 2006\end{array}$ & Coral recruitment $\downarrow$ \\
\hline \multicolumn{6}{|c|}{ Other benthic attributes: } \\
\hline \multirow[t]{2}{*}{ Diadema density } & Decrease & $\begin{array}{l}\text { Disease outbreak } \\
\text { Increase in predators }\end{array}$ & $\begin{array}{l}\text { Diadema disease } \\
\text { prevalence } \\
\text { Predator biomass } \\
\text { (principally species of } \\
\text { families Balistidae, } \\
\text { Diodontidae and } \\
\text { Haemulidae) }\end{array}$ & $\begin{array}{l}\text { Feehan and Scheibling, } \\
\text { 2014; Lessios, } 1988 \\
\text { Harborne et al., 2009; } \\
\text { McClanahan and Shafir, } \\
1990\end{array}$ & $\begin{array}{l}\text { Bioerosion } \downarrow \\
\text { Grazing } \downarrow\end{array}$ \\
\hline & Increase & $\begin{array}{l}\text { Reduction in predator } \\
\text { population }\end{array}$ & $\begin{array}{l}\text { Predator biomass } \\
\text { (principally species of } \\
\text { families Balistidae, }\end{array}$ & $\begin{array}{l}\text { Harborne et al., 2009; } \\
\text { McClanahan and Shafir, } \\
1990\end{array}$ & $\begin{array}{l}\text { Bioerosion } \uparrow \\
\text { Grazing } \uparrow\end{array}$ \\
\hline
\end{tabular}




\begin{tabular}{|c|c|c|c|c|c|}
\hline & & Increased recruitment & $\begin{array}{l}\text { Diodontidae and } \\
\text { Haemulidae) } \\
\text { Juvenile Diadema } \\
\text { density }\end{array}$ & $\begin{array}{l}\text { Carpenter and } \\
\text { Edmunds, } 2006\end{array}$ & \\
\hline Clionid sponge cover & Increase & $\begin{array}{l}\text { Decrease in water quality } \\
\text { Recent bleaching event }\end{array}$ & $\begin{array}{l}\text { Water quality data, } \\
\text { macroalgal cover, turf } \\
\text { canopy height, cover of } \\
\text { other benthic } \\
\text { heterotrophic feeders } \\
\text { (e.g. zoanthids) } \\
\text { Thermal anomaly data, } \\
\text { coral bleaching } \\
\text { incidence, coral partial } \\
\text { mortality }\end{array}$ & $\begin{array}{l}\text { Holmes, 2000; Rose and } \\
\text { Risk, 1985; Ward-Paige } \\
\text { et al., } 2005 \\
\text { Carballo et al., 2013; } \\
\text { Schönberg and Ortiz, } \\
2009\end{array}$ & $\begin{array}{l}\text { Bioerosion } \uparrow \\
\text { Bioerosion } \uparrow\end{array}$ \\
\hline $\begin{array}{l}\text { Sponge cover, } \\
\text { Sponge cover by } \\
\text { growth form, } \\
\text { Sponge height, } \\
\text { Sponge abundance } \\
\text { (excluding Clionids - } \\
\text { see separate attribute } \\
\text { above) }\end{array}$ & Decrease & $\begin{array}{l}\text { Sponge disease } \\
\text { Increased predation }\end{array}$ & $\begin{array}{l}\text { Sponge disease } \\
\text { incidence } \\
\text { Abundance of predators } \\
\text { (principally angelfishes) }\end{array}$ & $\begin{array}{l}\text { Webster, } 2007 \\
\text { Loh and Pawlik, 2014; } \\
\text { Pawlik et al., } 2013\end{array}$ & $\begin{array}{l}\text { Habitat provision } \downarrow \\
\text { (especially for massive } \\
\text { and erect branching } \\
\text { species) }\end{array}$ \\
\hline Octocoral cover, & Decrease & Octocoral disease & Octocoral disease & Harvell et al., 2007 & Habitat provision $\downarrow$ \\
\hline
\end{tabular}




\begin{tabular}{|c|c|c|c|c|c|}
\hline $\begin{array}{l}\text { Octocoral cover by } \\
\text { growth form }\end{array}$ & & & incidence & & \\
\hline Octocoral abundance & $\begin{array}{l}\text { Decrease in } \\
\text { phototrophic } \\
\text { octocorals and/ or } \\
\text { increase in } \\
\text { autotrophic } \\
\text { octocorals }\end{array}$ & $\begin{array}{l}\text { Increased water turbidity } \\
\text { and nutrients }\end{array}$ & $\begin{array}{l}\text { Water quality data, } \\
\text { sediment trap data }\end{array}$ & $\begin{array}{l}\text { De'ath and Fabricius, } \\
\text { 2010, } 2001\end{array}$ & Habitat provision $\downarrow$ \\
\hline $\begin{array}{l}\text { Cover of other sessile } \\
\text { organisms (e.g. } \\
\text { Corallimorphs, } \\
\text { Zoanthids) }\end{array}$ & Increase & Decreased water quality & $\begin{array}{l}\text { Water quality data, } \\
\text { macroalgal cover, turf } \\
\text { height, cover of other } \\
\text { benthic heterotrophic } \\
\text { feeders }\end{array}$ & $\begin{array}{l}\text { Cruz et al., 2014; } \\
\text { Kuguru et al., 2004; } \\
\text { Muhando et al., 2002; } \\
\text { Norström et al., } 2009\end{array}$ & Coral recruitment $\downarrow$ \\
\hline \multirow[t]{2}{*}{ Dead coral cover } & Acute increase & $\begin{array}{l}\text { Acute stressor }{ }^{\mathrm{a}} \text { causing } \\
\text { high coral mortality - most } \\
\text { be very recent as no algal } \\
\text { overgrowth of dead coral }\end{array}$ & $\begin{array}{l}\text { Coral cover, rugosity, } \\
\text { coral bleaching } \\
\text { incidence }\end{array}$ & Kramer, 2003 & $\begin{array}{l}\text { Coral recruitment } \downarrow \\
\text { Reef accretion } \downarrow\end{array}$ \\
\hline & Chronic increase & $\begin{array}{l}\text { Chronic stressor }^{\mathrm{b}} \text { causing } \\
\text { persistent coral mortality }\end{array}$ & $\begin{array}{l}\text { Coral cover by genus/ } \\
\text { species, disease/ } \\
\text { bleaching prevalence/ } \\
\text { incidence, coral growth } \\
\text { rate, macroalgal cover, } \\
\text { water quality data }\end{array}$ & $\begin{array}{l}\text { Chadwick and Morrow, } \\
2011\end{array}$ & $\begin{array}{l}\text { Coral recruitment } \downarrow \\
\text { Carbonate production } \\
\downarrow\end{array}$ \\
\hline Sediment cover & Increase & Storm redistributed & Water quality data, & Mah and Stearn, 1986; & Coral recruitment $\downarrow$ \\
\hline
\end{tabular}




\begin{tabular}{|c|c|c|c|c|c|}
\hline $\begin{array}{l}\text { (includes sand, mud, } \\
\text { silt and other fine } \\
\text { sediments) }\end{array}$ & & $\begin{array}{l}\text { sediments on the reef } \\
\text { Flooding event or } \\
\text { increased terrestrial run-off } \\
\text { smothered reef with silt } \\
\text { and other sediment }\end{array}$ & $\begin{array}{l}\text { sediment trap data, } \\
\text { coral cover, rugosity } \\
\text { Water quality data, } \\
\text { sediment trap data, } \\
\text { coral partial mortality }\end{array}$ & $\begin{array}{l}\text { Risk and Edinger, 2011; } \\
\text { Scoffin, } 1993 \\
\text { Bartley et al., 2013; } \\
\text { Fabricius, 2005; Risk } \\
\text { and Edinger, } 2011\end{array}$ & Coral growth $\downarrow$ \\
\hline Rubble cover & Increase & $\begin{array}{l}\text { Major mechanical stressor } \\
\text { (e.g. cyclones, ship } \\
\text { groundings, coral blasting) } \\
\text { fragmentation in situ or on } \\
\text { neighbouring reefs. } \\
\text { Bioerosion may assist } \\
\text { process }\end{array}$ & $\begin{array}{l}\text { Coral cover, rugosity, } \\
\text { clinoid sponge (and } \\
\text { other bioeroder) cover }\end{array}$ & $\begin{array}{l}\text { Fox and Caldwell, 2006; } \\
\text { Rasser and Riegl, 2002; } \\
\text { Scoffin, } 1993\end{array}$ & $\begin{array}{l}\text { Bioerosion } \uparrow \\
\text { Coral recruitment } \downarrow\end{array}$ \\
\hline $\begin{array}{l}\text { Bare substrate (rock, } \\
\text { pavement) cover }\end{array}$ & Increase & $\begin{array}{l}\text { Abrasion and erosion of } \\
\text { surfaces due to storm } \\
\text { Increased grazing intensity } \\
\text { by substrate excavators/ } \\
\text { scrapers (Urchins and } \\
\text { herbivores) }\end{array}$ & $\begin{array}{l}\text { Rubble cover, sediment } \\
\text { cover } \\
\text { Herbivore biomass } \\
\text { (herbivorous fish } \\
\text { excavators/scrapers and } \\
\text { Diadema) }\end{array}$ & $\begin{array}{l}\text { Mah and Stearn, 1986; } \\
\text { Woodley et al., } 1981 \\
\text { Williams et al., } 2001\end{array}$ & Coral recruitment $\uparrow$ \\
\hline Rugosity & Decrease & $\begin{array}{l}\text { Major mechanical stressor } \\
\text { (e.g. cyclones, ship } \\
\text { groundings, coral blasting) } \\
\text { damaged reef structure }\end{array}$ & $\begin{array}{l}\text { Coral cover, local } \\
\text { knowledge of recent } \\
\text { blasting/ ship } \\
\text { groundings and storms. }\end{array}$ & $\begin{array}{l}\text { Alvarez-Filip et al., } \\
\text { 2011; Bozec et al., 2015; } \\
\text { Fox and Caldwell, } 2006\end{array}$ & $\begin{array}{l}\text { Coral recruitment } \downarrow \\
\text { Habitat provision } \downarrow\end{array}$ \\
\hline
\end{tabular}




\begin{tabular}{|c|c|c|c|c|c|}
\hline & & $\begin{array}{l}\text { Increased bioerosion (most } \\
\text { likely driver if decrease in } \\
\text { rugosity is slow) }\end{array}$ & $\begin{array}{l}\text { Herbivore biomass, } \\
\text { cover of heterotrophic } \\
\text { feeders (e.g. clinoid } \\
\text { sponge cover), rubble } \\
\text { cover }\end{array}$ & $\begin{array}{l}\text { Alvarez-Filip et al., } \\
\text { 2011; Glynn, } 1997\end{array}$ & \\
\hline $\begin{array}{l}\text { Lobster (principally } \\
\text { Panulirus argus) } \\
\text { density }\end{array}$ & Decrease & $\begin{array}{l}\text { Increasing fishing pressure } \\
\text { Loss of habitat } \\
\text { Decrease in larval } \\
\text { recruitment } \\
\text { Disease outbreak }\end{array}$ & $\begin{array}{l}\text { Fisheries records and } \\
\text { size data (largest sizes } \\
\text { targeted by fishing) } \\
\text { Coral cover, rugosity } \\
\text { Lobster size-frequency } \\
\text { data } \\
\text { Disease incidence in } \\
\text { population }\end{array}$ & $\begin{array}{l}\text { Ehrhardt et al., 2011; } \\
\text { Wynne and Côté, } 2007 \\
\text { Ehrhardt et al., 2011; } \\
\text { Wynne and Côté, } 2007 \\
\text { Ehrhardt et al., } 2011 \\
\text { Behringer et al., } 2009\end{array}$ & \\
\hline $\begin{array}{l}\text { Conch (principally } \\
\text { Strombus gigas) } \\
\text { density }\end{array}$ & Decrease & $\begin{array}{l}\text { Increasing fishing pressure } \\
\text { Loss of habitat }\end{array}$ & $\begin{array}{l}\text { Fisheries record and size } \\
\text { data (largest sizes } \\
\text { targeted by fishing) } \\
\text { Seagrass and mangrove } \\
\text { survey data (key conch } \\
\text { habitats) }\end{array}$ & $\begin{array}{l}\text { Appeldoorn et al., 2011; } \\
\text { Stoner et al., } 2012\end{array}$ & \\
\hline $\begin{array}{l}\text { Crown-of-thorns } \\
\text { starfish density, Indo- } \\
\text { Pacific reefs only }\end{array}$ & Increase & Decreased water quality & $\begin{array}{l}\text { Water quality data, } \\
\text { macroalgal cover, turf } \\
\text { height, cover of other }\end{array}$ & $\begin{array}{l}\text { Brodie et al., 2005; } \\
\text { Fabricius et al., } 2010\end{array}$ & Bioerosion $\uparrow$ \\
\hline
\end{tabular}




\begin{tabular}{|c|c|c|c|c|}
\hline & & & $\begin{array}{l}\text { benthic heterotrophic } \\
\text { feeders }\end{array}$ & \\
\hline \multicolumn{5}{|l|}{ Fish: } \\
\hline $\begin{array}{l}\text { Abundance by species } \\
\& \\
\text { Total fish abundance }\end{array}$ & Decrease & Loss of habitat & $\begin{array}{l}\text { Fisheries data, species } \\
\text { level biomass data } \\
\text { Rugosity, coral cover }\end{array}$ & $\begin{array}{l}\text { Newman et al., 2006; } \\
\text { Wilson et al., } 2008 \\
\text { Gratwicke and Speight, } \\
\text { 2005; Wilson et al., } \\
2008\end{array}$ \\
\hline Length by species & Decrease & Potential overfishing & $\begin{array}{l}\text { Fisheries data, slope of } \\
\text { size-spectra }\end{array}$ & $\begin{array}{l}\text { Dulvy et al., 2004; } \\
\text { Graham et al., } 2005\end{array}$ \\
\hline $\begin{array}{l}\text { Biomass }{ }^{d} \text { by species } \\
\text { (see family groups } \\
\text { below for details) }\end{array}$ & Decrease & $\begin{array}{l}\text { Potential overfishing } \\
\text { Loss of habitat }\end{array}$ & $\begin{array}{l}\text { Fisheries data, species } \\
\text { level biomass data } \\
\text { Rugosity, coral cover }\end{array}$ & $\begin{array}{l}\text { Newman et al., 2006; } \\
\text { Wilson et al., } 2008 \\
\text { Gratwicke and Speight, } \\
\text { 2005; Wilson et al., } \\
2008\end{array}$ \\
\hline \multicolumn{5}{|l|}{ Biomass by familye: } \\
\hline $\begin{array}{ll}\text { - } & \text { Chaetodontidae } \\
\text { (Butterflyfishes) }\end{array}$ & Decrease & $\begin{array}{l}\text { Reduction in coral cover } \\
\text { (food and habitat) } \\
\text { Collection for aquarium } \\
\text { trade }\end{array}$ & $\begin{array}{l}\text { Cover cover, coral cover } \\
\text { by species, rugosity } \\
\text { Fisheries data }\end{array}$ & $\begin{array}{l}\text { Chong-Seng et al., 2012; } \\
\text { Cole et al., } 2008 \\
\text { Tissot and Hallacher, } \\
2003 \text {; Wabnitz et al., } \\
2003\end{array}$ \\
\hline
\end{tabular}


- Haemulidae

$$
\text { (Grunts) }
$$

- Lutjanidae

Decrease

(Snapper)

\&

- Serranidae (Groupers, Sea bass)

- Muraenidae (Moray eels)

Decrease
Overfishing

Decrease
Fisheries data,

abundance and length

data

Loss of nursery habitat

(mangroves and seagrass)

and/ or foraging grounds

Overfishing

Loss of reef complexity

Loss of nursery grounds

(mangroves, seagrasses and coral reef)

Overfishing

Loss of habitat

Habitat survey data

re

Fisheries data,

abundance and length

data

Rugosity, coral cover

Cora cover, rugosity, survey data

Fisheries data

Rugosity, coral cover seagrass and mangrove
Ault et al., 1998;

McClenachan and

Kittinger, 2013

Burke, 1995; Mumby et

al., 2004; Nagelkerken

et al., 2000

Hawkins and Roberts,

2004; Mumby et al.

2012

Gratwicke and Speight, 2005; Wilson et al.,

2008

Dorenbosch et al., 2005;

Igulu et al., 2014;

Mumby et al., 2004

Gilbert et al., 2005;

Johnson, 2010

Gilbert et al., 2005

Herbivorous fishes functional groups ${ }^{\mathrm{e}}$ biomass:
- Scrapers/
Decrease
Overfishing (fishing down
Fisheries data, turf
Edwards et al., 2014;
Grazing $\downarrow$ 


\begin{tabular}{|c|c|c|c|c|c|}
\hline excavators & & $\begin{array}{l}\text { the food web) } \\
\text { Loss of habitat }\end{array}$ & $\begin{array}{l}\text { canopy height } \\
\text { Rugosity, coral cover }\end{array}$ & $\begin{array}{l}\text { Mumby et al., } 2012 \\
\text { Bozec et al., 2013; } \\
\text { Wilson et al., } 2008\end{array}$ & Bioerosion $\downarrow$ \\
\hline & Increase & $\begin{array}{l}\text { Unless fishing pressure } \\
\text { recently declined, an } \\
\text { increase is usually } \\
\text { attributed to a large loss of } \\
\text { coral and increase in algal } \\
\text { food }\end{array}$ & $\begin{array}{l}\text { Coral cover, fisheries } \\
\text { data, turf canopy height }\end{array}$ & $\begin{array}{l}\text { Adam et al., 2011; } \\
\text { Gilmour et al., } 2013\end{array}$ & $\begin{array}{l}\text { Grazing } \uparrow \\
\text { Bioerosion } \uparrow\end{array}$ \\
\hline $\begin{array}{l}\text { - Grazers/ } \\
\text { detritivores }\end{array}$ & Decrease & $\begin{array}{l}\text { Overfishing (fishing down } \\
\text { the food web) } \\
\text { Loss of habitat }\end{array}$ & $\begin{array}{l}\text { Fisheries data, turf } \\
\text { canopy height } \\
\text { Rugosity, coral cover }\end{array}$ & $\begin{array}{l}\text { Edwards et al., 2014; } \\
\text { Mumby et al., } 2012 \\
\text { Bozec et al., 2013; } \\
\text { Wilson et al., } 2008\end{array}$ & Grazing $\downarrow$ \\
\hline - Browsers & Decrease & $\begin{array}{l}\text { Overfishing (fishing down } \\
\text { the food web) } \\
\text { Loss of habitat }\end{array}$ & $\begin{array}{l}\text { Fisheries data, } \\
\text { macroalgal cover } \\
\text { Rugosity, coral cover }\end{array}$ & $\begin{array}{l}\text { Edwards et al., 2014; } \\
\text { Mumby et al., } 2012 \\
\text { Bozec et al., 2013; } \\
\text { Wilson et al., } 2008\end{array}$ & Grazing $\downarrow$ \\
\hline $\begin{array}{l}\text { - Territorial } \\
\text { damselfish }\end{array}$ & Increase & $\begin{array}{l}\text { Decrease in abundance of } \\
\text { their predators (mainly } \\
\text { mesopredators) }\end{array}$ & $\begin{array}{l}\text { Damselfish predator } \\
\text { (mesopredators) } \\
\text { biomass, turf algae } \\
\text { cover }\end{array}$ & $\begin{array}{l}\text { Arnold et al., 2010; } \\
\text { Hoey and Bellwood, } \\
\text { 2009; Mumby et al., } \\
2012\end{array}$ & \\
\hline
\end{tabular}




\begin{tabular}{|c|c|c|c|c|}
\hline $\begin{array}{l}\text { Lionfish abundance, } \\
\text { Caribbean/ Western } \\
\text { Atlantic reefs only }\end{array}$ & Increase & $\begin{array}{l}\text { Lionfish population has not } \\
\text { reached maximum density }\end{array}$ & Biomass of prey species & $\begin{array}{l}\text { Green et al., 2012; } \\
\text { Morris and Akins, } 2009\end{array}$ \\
\hline
\end{tabular}

${ }^{a}$ Acute stressors: Storms (cyclones), coral blasting, shipwreck, mass bleaching event, major disease outbreak, Crown-of-thorns starfish outbreak, flooding or landslide resulting in heavy sedimentation, massive nutrient increase

${ }^{b}$ Chronic stressors: Continuous or repeated nutrient flow onto reef (e.g. from sewage leakage), continuous or repeated sediment flow onto reef (e.g. due to deforestation combined with rainfall), overfishing of herbivores (herbivorous fish and/ or Diadema)

${ }^{\mathrm{c}}$ Classified as autotrophic and heterotrophic for ecological interpretation

d Biomass is used rather than length or abundance because biomass can be interpreted more usefully and can be easily calculated from length and abundance values (Bohnsack and Harper, 1988).

e Biomass by family - family groups are those included in more than one monitoring programme. Herbivorous fishes (family groups Scaridae, Acanthuridae and Pomacanthidae) are broken into four functional groups (as per Edwards et al., 2014) so that ecologically meaningful interpretation can be provided. 


\subsection{Diagnosis of reef stressors}

The diagnostic decision tree is a representation of an elimination process for determining the most likely stressor(s) affecting a reef (Figure 2). It is a graphical summary of select indicators from Table 3 , and allows the path between reef health and possible stressors to be followed. Although all major reef stressors are included, the list of stressors is not exhaustive, so it is possible to make an incorrect diagnosis, just as with any diagnostic process.

A total of 14 indicators were used to construct the decision tree. Nine of these indicators stem from monitoring data: coral cover (used twice), coral cover by species, coral partial mortality, coral growth rate, macroalgal cover, turf algae height, rugosity, crown-of-thorns starfish density and herbivore biomass (used twice). Data for the other three indicators (recent storm, recent thermal anomaly, and proximal flooding or landslide) can be obtained through local knowledge or freely available internet databases (Table 1).

The diagnostic decision tree is split into two major branches: acute and chronic stressors. When an acute stressor has been diagnosed the diagnostic process is repeated, starting with the first indicator on the chronic stressor branch of the decision tree ("Increase in turf height?"), as an additional underlying, undiagnosed chronic stressor may also exist. Ideally, all indicators on the chronic stressor side of the diagnostic decision tree should be assessed to ensure no stressors are missed in the diagnosis, however this will depend on the data available to the user. In two cases an 'unknown' diagnosis may be reached. On the acute stressor branch of the tree, alternative explanations for this 'unknown' diagnosis include an unobserved coral predator outbreak or coral disease that caused coral loss across multiple species. Monitoring data on coral predator scars could be examined in the former case and adjacent reefs could be surveyed for disease in the latter case. For the 'unknown' diagnosis on the chronic stressor branch of the tree, coral predators or disease could also be the underlying cause, and appropriate monitoring data should be consulted.

If a diagnosis of 'Potential for future impact on reef' is reached, continued monitoring of herbivores and turf algal height is particularly important as declines in density or biomass of herbivores may lead to an increase in algal turf height or macroalgal cover, which could reduce coral recovery rates (Mumby et al., 2015) or even precede recruitment failure (Arnold and Steneck, 2011; Figure 1). 


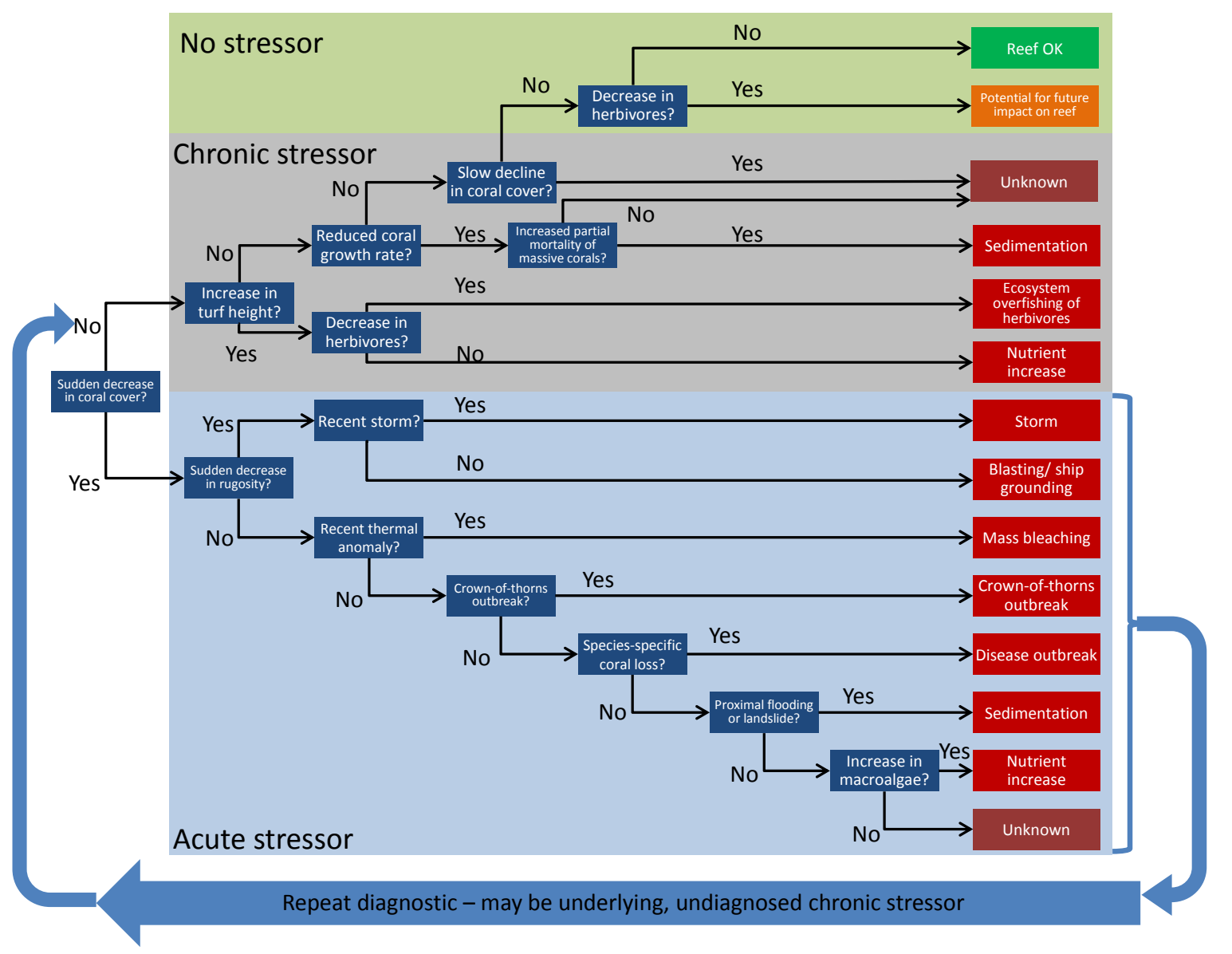

Figure 2. Diagnostic decision tree for main reef stressors. Stressors are shown in red boxes and indicators in blue boxes. When an acute stressor is diagnosed, the diagnostic processes should be repeated, starting with the "Increase in turf height?" indicator, to check for chronic stressors.

\subsection{Relative prognosis of reef health}

Several prognosis decision trees can be constructed depending on the stressors that were diagnosed. Two decision trees are presented here for the situations where the chronic stressors 'ecosystem overfishing of herbivores' or 'nutrient increase' have been diagnosed (Figure 3), and the acute stressors 'storms, coral blasting/ship grounding' or 'mass bleaching' have been diagnosed (Figure 4). Further decision trees are presented in the supplementary data (Figures A1 - 5 in Appendix A). For situations where both acute and chronic stressors have been diagnosed, the prognosis decision tree for the chronic stressor should be used as it is this stressor that will, by definition, have continuing impact on the reef and be more likely to affect the long-term prognosis.

By working through the indicators in the prognosis decision trees in turn, a prognosis score is obtained for a reef, with the lowest score representing the best prognosis. A reef's prognosis score places the reef on a scale from 'worst future scenario' to 'best future scenario'. These scores are not comparable between different decision trees and they do not represent absolute magnitudes of recovery potential.

In addition to being an accessible graphical representation of the prognosis scoring system, the decision trees also enable the user to identify the potential levers for management action in terms of 
improving the prognosis for a given reef. For example, in Figure 3, if a reef had a score of 6 (worst prognosis), management actions that could reduce macroalgal cover, such as increasing herbivore populations, would reduce that score to 4 , thus improving the relative prognosis outcome. Further information on the use of the prognosis decision trees is provided in Appendix B.

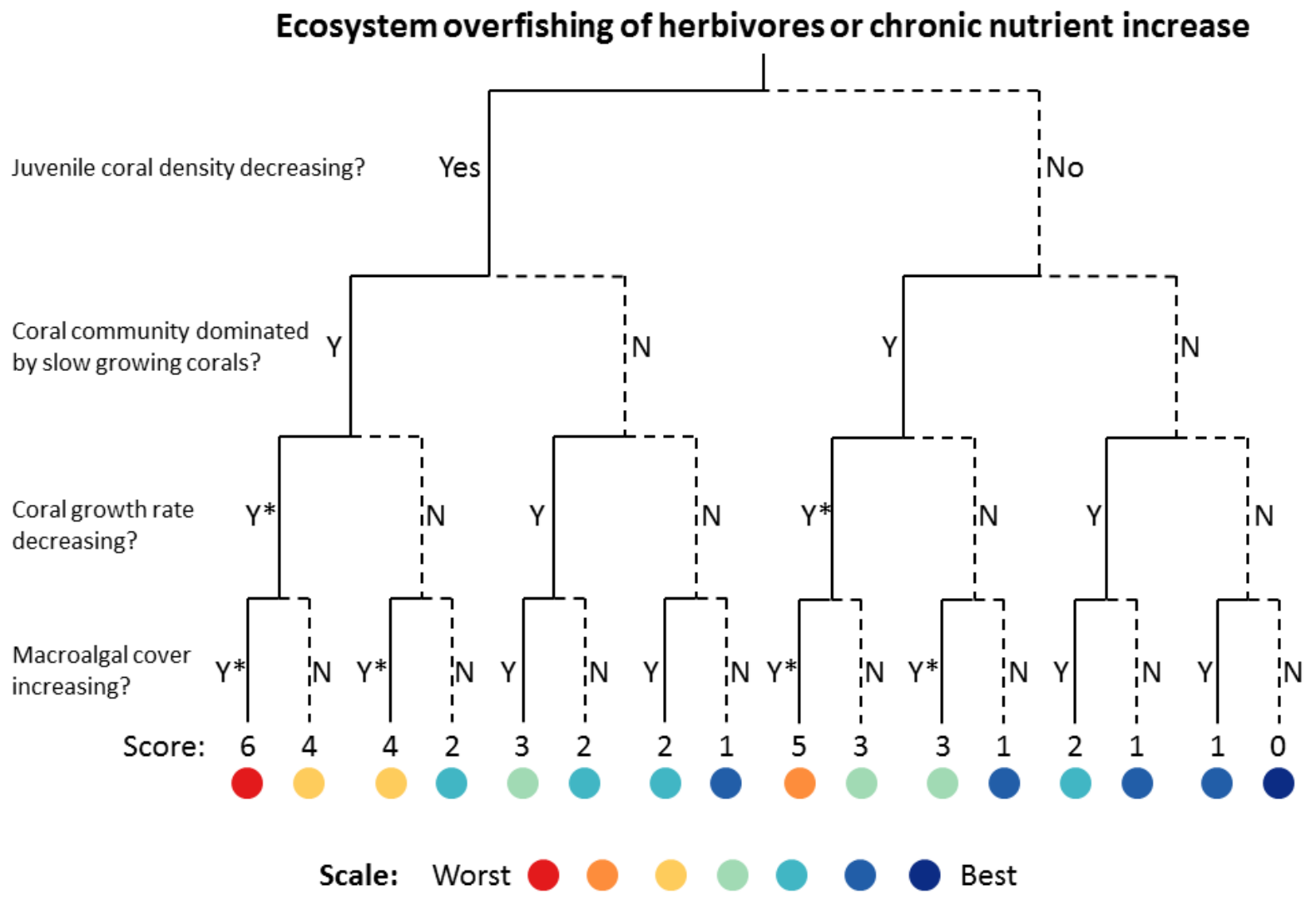

Figure 3. Prognosis decision tree for chronic stressors 'ecosystem overfishing of herbivores' or 'nutrient increase'. Dashed branches are for 'No' answer to indicators, solid branches for 'Yes' answers. Scores at the bottom of each branch are summation of scores obtained by working through each branch. Each 'Yes' answer ('Yes'/ ' $Y$ ') scores 1, each 'No' answer ('No'/ ' $N$ ') scores $\mathbf{0}$, and each Yes answer with asterisk (' $\left.\mathrm{Y}^{* \prime}\right)$ scores $\mathbf{2}$ to account for expected synergistic impacts. 


\section{Storm, blasting/ ship grounding or mass bleaching, and no chronic stressor}

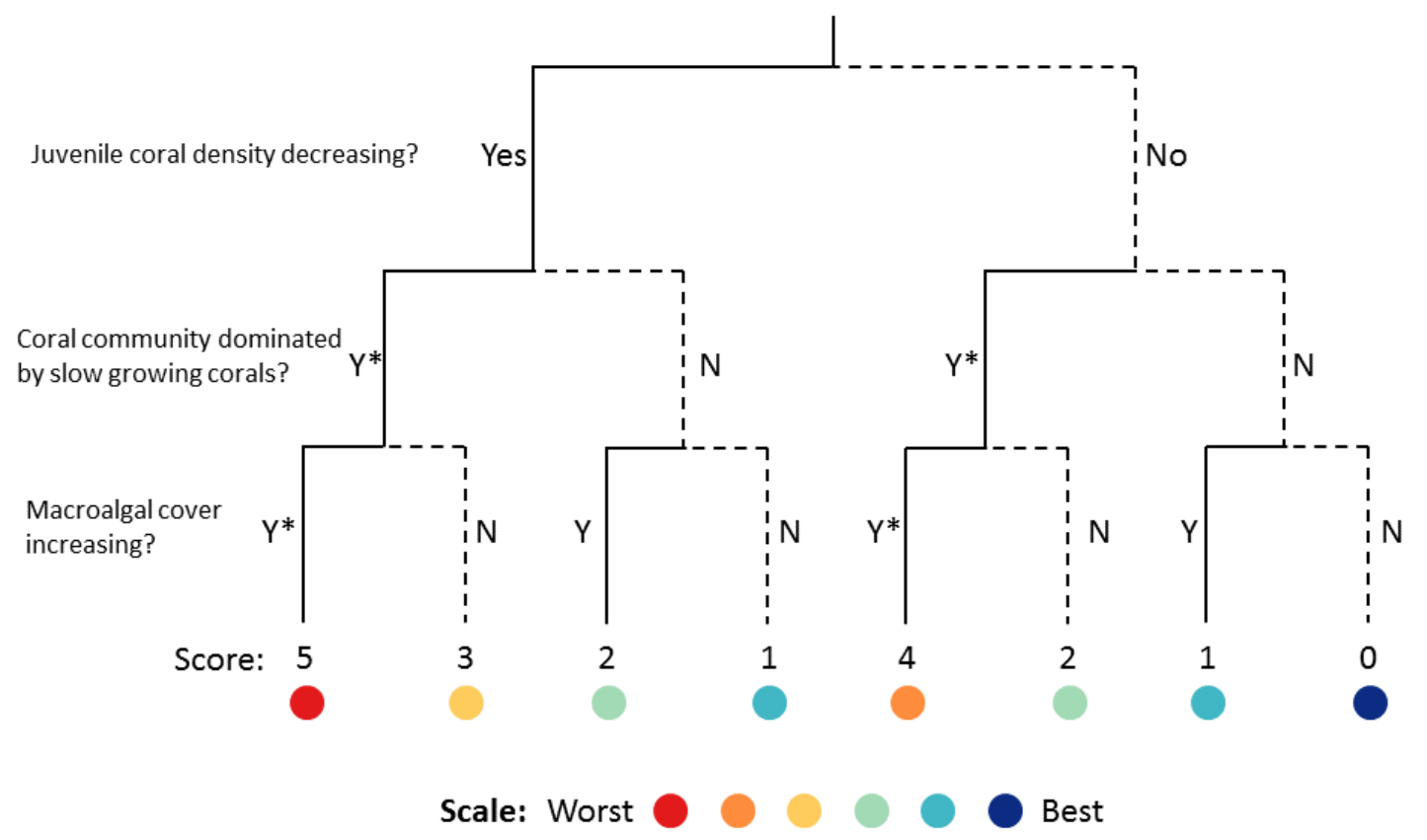

Figure 4. Prognosis decision tree for acute stressors 'storm', 'blasting/ship grounding' or 'mass bleaching', and where no chronic stressor has been diagnosed. Dashed branches are for 'No' answer to indicators, solid branches for 'Yes' answers. Scores at the bottom of each branch are summation of scores obtained by working through each branch. Each 'Yes' answer ('Yes'/ ' $Y$ ') scores 1, each 'No' answer ('No'/ ' $N$ ') scores 0 , and each Yes answer with asterisk (' $\left.\mathrm{F}^{* \prime}\right)$ scores 2 to account for expected synergistic impacts.

\subsection{Informing management decisions}

Once a stressor has been diagnosed, it allows management actions to focus on the driver of that stressor where possible. For example, if the diagnosis reveals sedimentation as the stressor, management can focus on controlling sources of sediment, possibly through watershed management or control of coastal development. However, for many of the acute stressors, such as storms or mass bleaching, it will not be possible to manage the immediate drivers of the stressor. In these cases management actions can focus on improving preparedness for mitigating future stressors, work to reduce any chronic stressors to the reef, and, in some cases, assist recovery through restoration efforts (Table 4).

Table 4. Potential management actions for reef stressors

\begin{tabular}{|c|c|c|}
\hline Stressor & Acute/ Chronic & $\begin{array}{l}\text { Potential management actions (partially adapted } \\
\text { from Anthony et al., 2014) }\end{array}$ \\
\hline Sedimentation & Both & $\begin{array}{l}\text { Improve management of watershed through } \\
\text { education, regulation, incentives and penalties. Re- } \\
\text { vegetation of surrounding land. Control coastal }\end{array}$ \\
\hline
\end{tabular}




\begin{tabular}{|c|c|c|}
\hline & & development activities \\
\hline $\begin{array}{l}\text { Ecosystem overfishing of } \\
\text { herbivores }\end{array}$ & Chronic & $\begin{array}{l}\text { Improve fisheries management through education, } \\
\text { regulation, incentives and penalties. For example: } \\
\text { ban trap fishing and/ or herbivore harvesting, } \\
\text { implement no-take zones }\end{array}$ \\
\hline Nutrient increase & Both & $\begin{array}{l}\text { Improve management of urban, agricultural and } \\
\text { shipping activities through education, regulation, } \\
\text { incentives and penalties }\end{array}$ \\
\hline Storm & Acute & $\begin{array}{l}\text { Preparedness and recovery planning locally. Reduce } \\
\text { any local chronic stressors }\end{array}$ \\
\hline Coral blasting & Acute & $\begin{array}{l}\text { Increase incentives for non-destructive harvest of } \\
\text { resource through education, regulation and } \\
\text { enforcement. Improve recovery through substrate } \\
\text { stabilisation and reef restoration measures }\end{array}$ \\
\hline Ship grounding & Acute & $\begin{array}{l}\text { Reduce likelihood of groundings through education, } \\
\text { regulation, incentives and penalties. Use } \\
\text { compensation payments to aid recovery through } \\
\text { substrate stabilisation and reef restoration measures }\end{array}$ \\
\hline Mass coral bleaching & Acute & $\begin{array}{l}\text { Reduce any local chronic stressors. Identify sites that } \\
\text { may have lower vulnerability as possible refugia for } \\
\text { protection }\end{array}$ \\
\hline $\begin{array}{l}\text { Crown-of-thorns starfish } \\
\text { outbreak }\end{array}$ & Acute & $\begin{array}{l}\text { Improve management of watershed through } \\
\text { education, regulation, incentives and penalties. } \\
\text { Protection of CoTS predators, surveillance to detect } \\
\text { early signs of CoTS build up with tactical CoTS control }\end{array}$ \\
\hline Disease outbreak & Acute & $\begin{array}{l}\text { Reduce stressors that can increase incidence and } \\
\text { severity of diseases such as increased nutrient levels } \\
\text { and other land-based runoff (see sedimentation and } \\
\text { nutrient increase above) }\end{array}$ \\
\hline
\end{tabular}

\subsection{Potential improvements to monitoring programmes}

The indicators used in diagnosis and prognosis can form a minimal set to be included in reef monitoring programmes (Table 5). Two of these indicators are not commonly included in monitoring programmes: turf canopy height is measured in only two of the programmes we collated data from, and coral growth rate in only one. Their inclusion in all programmes might increase the management usefulness of monitoring results. Turf canopy height is a rapid indicator of a shift in the relative importance of bottom-up and top-down controls of algae, thereby indicating either a strengthening of nutrification or a reduction in herbivory. Experimental and observational studies have found that 
an increase in turf canopy height can reduce coral recruitment directly (Arnold et al., 2010; Mumby et al., 2013) and the situation is compounded if turfs also trap sediments (Birrell et al., 2008).

Measuring algal turf height is a quick and simple process requiring only a ruler, and is therefore easy to include in reef monitoring programmes. Coral colony growth rate can reflect the impacts of multiple stressors including sediments, temperature, and algal competition (Box and Mumby, 2007; Carricart-Ganivet and Merino, 2001; Carricart-Ganivet et al., 2012) and strongly influences the ability of a reef to recover after an impact (Ortiz et al., 2014a). Growth rate can be measured using permanent quadrats (van Woesik et al., 2009) or tagging individual colonies (English et al., 1997). Tags can be attached using plastic cable ties for branching corals or nails for massive colonies (Hill and Wilkinson, 2004), though the use of nails may be prohibited in some protected areas and can be damaging to the coral.

Table 5. Key indicators used in diagnosis and prognosis

\begin{tabular}{|l|l|l|}
\hline Indicator & Used in: & \\
& Diagnosis? & Prognosis? \\
\hline Coral cover & $\checkmark$ & $\checkmark$ \\
\hline Coral cover by species & $\checkmark$ & $\checkmark$ \\
\hline Juvenile coral density & & $\checkmark$ \\
\hline Coral growth rate & $\checkmark$ & $\checkmark$ \\
\hline Coral partial mortality & $\checkmark$ & $\checkmark$ \\
\hline Coral disease incidence & & $\checkmark$ \\
\hline Macroalgae cover & $\checkmark$ & $\checkmark$ \\
\hline Turf canopy height & $\checkmark$ & $\checkmark$ \\
\hline Crown-of-thorns starfish density & $\checkmark$ & \\
\hline $\begin{array}{l}\text { Herbivore biomass (fish and Diadema where they are } \\
\text { significant grazers) }\end{array}$ & $\checkmark$ & \\
\hline Rugosity & & $\checkmark$ \\
\hline
\end{tabular}

Disease prevalence was measured by all except one of the reef monitoring programmes, but none of them measured disease incidence which we have used in the prognosis. Prevalence is the proportion of diseased corals at a single point in time, whereas incidence is the number of new cases of disease over time. Prevalence data are easier to collect on a snapshot survey but have limited value for monitoring. For example, the coral population might have a constant $10 \%$ prevalence of diseased colonies but the implications are dramatically different if this $10 \%$ constitutes the same colonies from year to year (i.e. corals that tolerate the disease) versus a new set of infected colonies each year which likely implies that coral populations will decline markedly over time. Resolving the latter requires tagging of colonies (both infected and uninfected) and monitoring changes in the incidence as well as the response of corals to disease. Although not included in diagnosis or prognosis, bleaching incidence can also be a useful indicator.

Monitoring needs to be done at appropriate time intervals to detect trends with sufficient early warning to affect useful management interventions. Due to economic and logistic factors, there is a trade-off between the frequency of monitoring and the number of sites that can be sampled. For example, frequent sampling at a small number of sites may not give a true picture of overall reef health in an area, but could still be time consuming and expensive (Hill and Wilkinson, 2004). For many reefs, annual or even biennial monitoring is sufficient to detect change, as many changes are 
slow, e.g. the Great Barrier Reef Long -term Monitoring Program uses both broad-scale annual surveys and biennial intensive surveys to balance the need for detail and spatial and temporal coverage (Sweatman et al. 2008). However, monitoring should also be adaptive so that following an acute stressor, such as a bleaching event, more frequent monitoring, possibly including more sites, can be initiated to track reef recovery or decline.

\subsection{Limitations of methods and future directions}

The methods presented here are a first attempt at providing a general framework for interpreting reef monitoring data. However, the relative importance of ecological processes varies geographically; therefore the framework would benefit from careful tailoring to specific regions. For example, herbivory in the Caribbean is currently dominated by parrotfishes (Mumby et al., 2006) whereas surgeonfishes likely dominate this function in many Pacific locations (Marshell and Mumby, 2015; Russ, 1984). Furthermore, as work continues on finding ecosystem thresholds (e.g. Karr et al., 2015), these can be incorporated into the methods. In other words, the principles embodied in the framework are likely to hold generically although the specific details and thresholds will differ from region to region.

As many reefs are currently impacted by more than one stressor (Ban et al., 2014), an improvement to the methods developed here would be to evaluate whether multiple stressors act additively, synergistically, or even as an antagonism. While both empirical (Darling et al., 2010) and modelling studies (Bozec and Mumby, 2015) have looked at the impacts of multiple stressors, more work will be needed before a general framework for understanding and quantifying these impacts can be provided. Long-term monitoring of reefs will help in this process, providing the opportunity to study multiple stressors, which more reefs will be exposed to as the impacts of climate change increase in frequency and intensity (Côté et al., 2016).

\section{Conclusion}

Reef monitoring is expensive and there have been previous criticisms of the lack of focus on useful indicators (Downs et al., 2005; Risk, 1999). Calls to change the suite of attributes recorded on surveys have generally gone unheeded, most likely because of difficulty prioritizing attributes given the great complexity of the reef ecosystem, historical inertia of programmes, and confusion and disagreement as to the best indicators. Our attempt to build a framework for the interpretation of monitoring data draws on a diverse ecological literature and we hope that continued advances in reef science can be incorporated to improve such frameworks in future. While our framework should not surprise reef ecologists, we hope that it will help practitioners interpret monitoring data and identify appropriate management actions in the field.

\section{Acknowledgements}

This article was funded by the European Union Seventh Framework Programme (P7/2007-2013) under grant agreement no. 244161 (Future of Reefs in a Changing Environment), an SIEF fellowship to $\mathrm{KH}$ and an ARC Laureate Fellowship to PJM. Vector graphics in Figure 1 and Graphical Abstract courtesy of the Integration and Application Network, University of Maryland Center for Environmental Science (ian.umces.edu/symbols/), and Alice Rogers. We are grateful to Heron Island Research Station for hosting the workshop that led to this paper and our colleagues at the Marine 
Spatial Ecology Lab for productive discussions. We thank two referees for their helpful comments on the manuscript.

\section{References}

Adam, T.C., Schmitt, R.J., Holbrook, S.J., Brooks, A.J., Edmunds, P.J., Carpenter, R.C., Bernardi, G., 2011. Herbivory, Connectivity, and Ecosystem Resilience: Response of a Coral Reef to a LargeScale Perturbation. PLoS One 6, e23717. doi:10.1371/journal.pone.0023717

Albert, S., O'Neil, J.M., Udy, J.W., Ahern, K.S., O'Sullivan, C.M., Dennison, W.C., 2005. Blooms of the cyanobacterium Lyngbya majuscula in coastal Queensland, Australia: disparate sites, common factors. Mar. Pollut. Bull. 51, 428-437. doi:10.1016/j.marpolbul.2004.10.016

Alvarado-Chacón, E.M., Acosta, A., 2009. Population Size-Structure of the Reef- Coral Montastraea Annularis in Two Contrasting Reefs of a Marine Protected Area in the Southern Caribbean Sea. Bull. Mar. Sci. 85, 61-76.

Alvarez-Filip, L., Gill, J.A., Dulvy, N.K., Perry, A.L., Watkinson, A.R., Côté, I.M., 2011. Drivers of regionwide declines in architectural complexity on Caribbean reefs. Coral Reefs 30, 1051-1060. doi:10.1007/s00338-011-0795-6

Anthony, K.R., Marshall, P.A., Abdulla, A., Beeden, R., Bergh, C., Black, R., Eakin, C.M., Game, E.T., Gooch, M., Graham, N.A.J., Green, A., Heron, S.F., van Hooidonk, R., Knowland, C., Mangubhai, S., Marshall, N., Maynard, J.A., McGinnity, P., McLeod, E., Mumby, P.J., Nyström, M., Obura, D., Oliver, J., Possingham, H.P., Pressey, R.L., Rowlands, G.P., Tamelander, J., Wachenfeld, D., Wear, S., 2014. Operationalizing resilience for adaptive coral reef management under global environmental change. Glob. Chang. Biol. 21, 48 - 61. doi:10.1111/gcb.12700

Appeldoorn, R.S., Gonzalez, E.C., Glazer, R., Prada, M., 2011. Applying EBM to Queen conch fisheries in the Caribbean, in: Fanning, L., Mahon, R., McConney, P. (Eds.), Towards Ecosystem-Based Management in the Wider Caribbean. Amsterdam University Press, Amsterdam, pp. 177-186.

Arnold, S.N., Steneck, R.S., 2011. Settling into an Increasingly Hostile World: The Rapidly Closing "Recruitment Window" for Corals. PLoS One 6, e28681. doi:10.1371/journal.pone.0028681

Arnold, S.N., Steneck, R.S., Mumby, P.J., 2010. Running the gauntlet: inhibitory effects of algal turfs on the processes of coral recruitment. Mar. Ecol. Prog. Ser. 414, 91-105. doi:10.3354/meps08724

Aronson, R.B., Precht, W.F., 2001. White-band disease and the changing face of Caribbean coral reefs. Hydrobiologia 460, 25-38. doi:10.1023/A:1013103928980

Ault, J.S., Bohnsack, J.A., Meester, G.A., 1998. A retrospective (1979-1996) multispecies assessment of coral reef fish stocks in the Florida Keys. Fish. Bull. 96, 395-414.

Baird, A.H., Marshall, P.A., 2002. Mortality, growth and reproduction in scleractinian corals following bleaching on the Great Barrier Reef. Mar. Ecol. Prog. Ser. 237, 133-141. doi:10.3354/meps237133

Ban, S.S., Graham, N.A.J., Connolly, S.R., 2014. Evidence for multiple stressor interactions and effects on coral reefs. Glob. Chang. Biol. 20, 681-697. doi:10.1111/gcb.12453

Bartley, R., Bainbridge, Z.T., Lewis, S.E., Kroon, F.J., Wilkinson, S.N., Brodie, J.E., Silburn, D.M., 2013. Relating sediment impacts on coral reefs to watershed sources, processes and management: $\mathrm{A}$ review. Sci. Total Environ. 468-469, 1138-1153. doi:10.1016/j.scitotenv.2013.09.030

Behringer, D., IV, M.B., Shields, J., 2009. A review of the lethal spiny lobster virus PaV 1- Ten years 
after its discovery, in: Proceedings of the 62nd Gulf and Caribbean Fisheries Institute, Cumana, Venezuela, pp. $370-375$.

Birrell, C.L., Mccook, L.J., Willis, B.L., Diaz-Pulido, G.A., 2008. Effects of benthic algae on the replenishment of corals and the implications for the resilience of coral reefs. Oceanogr. Mar. Biol. An Annu. Rev. 46, 25-63. doi:10.1201/9781420065756.ch2

Bohnsack, J.A., Bannerot, S.P., 1986. A stationary visual census technique for quantitatively assessing community structure of coral reef fishes, NOAA Technical Report NMFS-41, National Oceanic and Atmospheric Administration, Miami

Bohnsack, J.A., Harper, D.E., 1988. Length-weight relationships of selected marine reef fishes from the southeastern United States and the Caribbean. NOAA Technical Memorandum NMFS-SEFC215, National Oceanic and Atmospheric Administration, Miami

Borja, A., Dauer, D.M., 2008. Assessing the environmental quality status in estuarine and coastal systems: Comparing methodologies and indices. Ecol. Indic. 8, 331-337. doi:http://dx.doi.org/10.1016/j.ecolind.2007.05.004

Box, S.J., Mumby, P.J., 2007. Effect of macroalgal competition on growth and survival of juvenile Caribbean corals. Mar. Ecol. Prog. Ser. 342, 139-149. doi:10.3354/meps342139

Bozec, Y.-M., Alvarez-Filip, L., Mumby, P.J., 2015. The dynamics of architectural complexity on coral reefs under climate change. Glob. Chang. Biol. 21, 223-235. doi:10.1111/gcb.12698

Bozec, Y.-M., Mumby, P.J., 2015. Synergistic impacts of global warming on the resilience of coral reefs. Philos. Trans. R. Soc. B Biol. Sci. 370, 20130267. doi:10.1098/rstb.2013.0267

Bozec, Y.-M., Yakob, L., Bejarano, S., Mumby, P.J., 2013. Reciprocal facilitation and non-linearity maintain habitat engineering on coral reefs. Oikos 122, 428-440. doi:10.1111/j.1600-

0706.2012.20576.x

Bradley, P., Fisher, W.S., Bell, H., Davis, W., Chan, V., LoBue, C., Wiltse, W., 2008. Development and implementation of coral reef biocriteria in U.S. jurisdictions. Environ. Monit. Assess. 150, 4351. doi:10.1007/s10661-008-0670-2

Brocke, H.J., Polerecky, L., de Beer, D., Weber, M., Claudet, J., Nugues, M.M., 2015. Organic Matter Degradation Drives Benthic Cyanobacterial Mat Abundance on Caribbean Coral Reefs. PLoS One 10, e0125445. doi:10.1371/journal.pone.0125445

Brodie, J., Fabricius, K.E., De'ath, G., Okaji, K., 2005. Are increased nutrient inputs responsible for more outbreaks of crown-of-thorns starfish? An appraisal of the evidence. Mar. Pollut. Bull. 51, 266-278. doi:10.1016/j.marpolbul.2004.10.035

Brown, B.E., 1997. Coral bleaching : causes and consequences. Coral Reefs 16, 129-138. doi:10.1007/s003380050249

Brown, E., Cox, E., Jokiel, P., Rodgers, K., Smith, W., Tissot, B., Coles, S.L., Hultquist, J., 2004. Development of Benthic Sampling Methods for the Coral Reef Assessment and Monitoring Program (CRAMP) in Hawai'i. Pacific Sci. 58, 145-158. doi:10.1353/psc.2004.0013

Burke, N.C., 1995. Nocturnal foraging habitats of French and bluestriped grunts, Haemulon flavolineatum and H. sciurus, at Tobacco Caye, Belize. Environ. Biol. Fishes 42, 365-374. doi:10.1007/BF00001467

Burkepile, D.E., Hay, M.E., 2009. Nutrient versus herbivore control of macroalgal community development and coral growth on a Caribbean reef. Mar. Ecol. Prog. Ser. 389, 71-84. doi:10.3354/meps08142 
Burkepile, D.E., Hay, M.E., 2006. Herbivore vs. nutrient control of marine primary producers: Context-dependent effects. Ecology 87, 3128-3139. doi:10.1890/00129658(2006)87[3128:HVNCOM]2.0.CO;2

Carballo, J.L., Bautista, E., Nava, H., Cruz-Barraza, J.A., Chávez, J.A., 2013. Boring sponges, an increasing threat for coral reefs affected by bleaching events. Ecol. Evol. 3, 872-886. doi:10.1002/ece3.452

CaribNode, 2016. www.caribnode.org/ [WWW Document]. URL http://www.caribnode.org/ (accessed 8.14.16).

CARICOMP, 2001. The Caribbean Coastal Marine Productivity Program (CARICOMP). Bull. Mar. Sci. $69,819-829$.

Carleton, J.H., Done, T.J., 1995. Quantitative video sampling of coral reef benthos: large-scale application. Coral Reefs 14, 35-46. doi:10.1007/BF00304070

Carpenter, R.C., Edmunds, P.J., 2006. Local and regional scale recovery of Diadema promotes recruitment of scleractinian corals. Ecol. Lett. 9, 271-280. doi:10.1111/j.14610248.2005.00866.x

Carricart-Ganivet, J.P., Cabanillas-Terán, N., Cruz-Ortega, I., Blanchon, P., 2012. Sensitivity of calcification to thermal stress varies among genera of massive reef-building corals. PLoS One 7 , e32859. doi:10.1371/journal.pone.0032859

Carricart-Ganivet, J.P., Merino, M., 2001. Growth responses of the reef-building coral Montastraea annularis along a gradient of continental influence in the southern Gulf of Mexico. Bull. Mar. Sci. $68,133-146$.

Chabanet, P., Adjeroud, M., Andréfouët, S., Bozec, Y.-M., Ferraris, J., Garcìa-Charton, J.-A., Schrimm, M., 2005. Human-induced physical disturbances and their indicators on coral reef habitats: $A$ multi-scale approach. Aquat. Living Resour. 18, 215-230. doi:10.1051/alr:2005028

Chadwick, N.E., Morrow, K.M., 2011. Competition Among Sessile Organisms on Coral Reefs, in: Dubinsky, Z., Stambler, N. (Eds.), Coral Reefs: An Ecosystem in Transition. Springer, pp. 347371. doi:10.1007/978-94-007-0114-4_20

Chong-Seng, K.M., Mannering, T.D., Pratchett, M.S., Bellwood, D.R., Graham, N. a J., 2012. The influence of coral reef benthic condition on associated fish assemblages. PLoS One 7, e42167. doi:10.1371/journal.pone.0042167

Cole, A.J., Pratchett, M.S., Jones, G.P., 2008. Diversity and functional importance of coral-feeding fishes on tropical coral reefs. Fish Fish. 9, 286-307. doi:10.1111/j.1467-2979.2008.00290.x

Cooper, T.F., Gilmour, J.P., Fabricius, K.E., 2009. Bioindicators of changes in water quality on coral reefs: review and recommendations for monitoring programmes. Coral Reefs 28, 589-606. doi:10.1007/s00338-009-0512-x

Cortés, J., Risk, M.J., 1985. A reef under siltation stress: Cahuita, Costa Rica. Bull. Mar. Sci. 36, 339356.

Côté, I.M., Darling, E.S., Brown, C.J., 2016. Interactions among ecosystem stressors and their importance in conservation. Proc. R. Soc. London B Biol. Sci. 283, 20152592. doi:10.1098/rspb.2015.2592

Couch, C.S., Garriques, J.D., Barnett, C., Preskitt, L., Cotton, S., Giddens, J., Walsh, W., 2014. Spatial and temporal patterns of coral health and disease along leeward Hawai'i Island. Coral Reefs 33, 693-704. doi:10.1007/s00338-014-1174-x 
Cruz, I.C.S., de Kikuchi, R.K.P., Longo, L.L., Creed, J.C., 2014. Evidence of a phase shift to Epizoanthus gabrieli Carlgreen, 1951 (Order Zoanthidea) and loss of coral cover on reefs in the Southwest Atlantic. Mar. Ecol. doi:10.1111/maec.12141

Darling, E.S., McClanahan, T.R., Côté, I.M., 2010. Combined effects of two stressors on Kenyan coral reefs are additive or antagonistic, not synergistic. Conserv. Lett. 3, 122-130. doi:10.1111/j.1755-263X.2009.00089.x

Day, J., 2008. The need and practice of monitoring, evaluating and adapting marine planning and management - lessons from the Great Barrier Reef. Mar. Policy 32, 823-831. doi:10.1016/j.marpol.2008.03.023

De'ath, G., Fabricius, K.E., 2010. Water quality as a regional driver of coral biodiversity and macroalgae on the Great Barrier Reef. Ecol. Appl. 20, 840-850. doi:10.1890/08-2023.1

Diaz-Pulido, G.A., McCook, L.J., 2002. The fate of bleached corals: Patterns and dynamics of algal recruitment. Mar. Ecol. Prog. Ser. 232, 115-128. doi:10.3354/meps232115

Diaz-Pulido, G.A., McCook, L.J., Dove, S., Berkelmans, R., Roff, G., Kline, D.I., Weeks, S., Evans, R.D., Williamson, D.H., Hoegh-Guldberg, O., 2009. Doom and Boom on a Resilient Reef: Climate Change, Algal Overgrowth and Coral Recovery. PLoS One 4, e5239. doi:10.1371/journal.pone.0005239

Dorenbosch, M., Grol, M.G.G., Christianen, M.J.A., Nagelkerken, I., Van Der Velde, G., 2005. IndoPacific seagrass beds and mangroves contribute to fish density and diversity on adjacent coral reefs. Mar. Ecol. Prog. Ser. 302, 63-76. doi:10.3354/meps302063

Downs, C.A., Ostrander, G.K., Rougee, L., Rongo, T., Knutson, S., Williams, D.E., Mendiola, W., Holbrook, J., Richmond, R.H., 2012. The use of cellular diagnostics for identifying sub-lethal stress in reef corals. Ecotoxicology 21, 768-782. doi:10.1007/s10646-011-0837-4

Downs, C.A., Woodley, C.M., Richmond, R.H., Lanning, L.L., Owen, R., 2005. Shifting the paradigm of coral-reef "health" assessment. Mar. Pollut. Bull. 51, 486-94.

doi:10.1016/j.marpolbul.2005.06.028

Dulvy, N.K., Polunin, N.V.C., Mill, A.C., Graham, N.A.J., 2004. Size structural change in lightly exploited coral reef fish communities : evidence for weak indirect effects. Can. J. Fish. Aquat. Sci. 61, 466-475. doi:10.1139/F03-169

Eakin, C.M., Morgan, J.A., Heron, S.F., Smith, T.B., Liu, G., Alvarez-Filip, L., Baca, B., Bartels, E., Bastidas, C., Bouchon, C., Brandt, M., Bruckner, A.W., Bunkley-Williams, L., Cameron, A., Causey, B.D., Chiappone, M., Christensen, T.R.L., Crabbe, M.J.C., Day, O., de la Guardia, E., DíazPulido, G., DiResta, D., Gil-Agudelo, D.L., Gilliam, D.S., Ginsburg, R.N., Gore, S., Guzmán, H.M., Hendee, J.C., Hernández-Delgado, E.A., Husain, E., Jeffrey, C.F.G., Jones, R.J., Jordán-Dahlgren, E., Kaufman, L.S., Kline, D.I., Kramer, P.A., Lang, J.C., Lirman, D., Mallela, J., Manfrino, C., Maréchal, J.-P., Marks, K., Mihaly, J., Miller, W.J., Mueller, E.M., Muller, E.M., Orozco Toro, C.A., Oxenford, H.A., Ponce-Taylor, D., Quinn, N., Ritchie, K.B., Rodríguez, S., Ramírez, A.R., Romano, S., Samhouri, J.F., Sánchez, J. a, Schmahl, G.P., Shank, B. V, Skirving, W.J., Steiner, S.C.C., Villamizar, E., Walsh, S.M., Walter, C., Weil, E., Williams, E.H., Roberson, K.W., Yusuf, Y., 2010. Caribbean corals in crisis: record thermal stress, bleaching, and mortality in 2005. PLoS One 5, e13969. doi:10.1371/journal.pone.0013969

Edwards, A.J., Gomez, E.D., 2007. Reef restoration concepts and guidelines: making sensible management choices in the face of uncertainty. Coral Reef Targeted Research \& Capacity Building for Management Programme: St Lucia, Australia. iv + 38 pp

Edwards, C., Friedlander, A.M., Green, A.G., Hardt, M.J., Sala, E., Sweatman, H., Williams, I.D., 
Zgliczynski, B.J., Sandin, S.A., Smith, J.E., 2014. Global assessment of the status of coral reef herbivorous fishes: evidence for fishing effects. Proc. R. Soc. B Biol. Sci. 281.

doi:10.1098/rspb.2013.1835

Ehrhardt, N., Puga, R., Butler, M.I., 2011. Implications of the ecosystem approach to fisheries management in large ecosystems: The case of the Caribbean spiny lobster, in: Fanning, L., Mahon, R., McConney, P. (Eds.), Towards Ecosystem-Based Management in the Wider Caribbean. Amsterdam University Press, Amsterdam, pp. 157 - 176.

English, S., Wilkinson, C.R., Baker, V., 1997. Survey manual for tropical marine resources. Australian Institute of Marine Science, Townsville, Australia.

Fabricius, K.E., 2005. Effects of terrestrial runoff on the ecology of corals and coral reefs: review and synthesis. Mar. Pollut. Bull. 50, 125-46. doi:10.1016/j.marpolbul.2004.11.028

Fabricius, K.E., De'ath, G., 2001. Biodiversity on the Great Barrier Reef: large-scale patterns and turbidity-related local loss of soft coral taxa, in: Wolanski, E. (Ed.), Oceanographic Processes of Coral Reefs : Physical and Biological Links in the Great Barrier Reef. CRC Press, Boca Raton, Florida, pp. 127-144.

Fabricius, K.E., Okaji, K., De'ath, G., 2010. Three lines of evidence to link outbreaks of the crown-ofthorns seastar Acanthaster planci to the release of larval food limitation. Coral Reefs 29, 593605. doi:10.1007/s00338-010-0628-z

Feehan, C.J., Scheibling, R.E., 2014. Effects of sea urchin disease on coastal marine ecosystems. Mar. Biol. 161, 1467-1485. doi:10.1007/s00227-014-2452-4

Ferrari, R., Gonzalez-Rivero, M., Ortiz, J.C., Mumby, P.J., 2012. Interaction of herbivory and seasonality on the dynamics of Caribbean macroalgae. Coral Reefs 31, 683-692. doi:10.1007/s00338-012-0889-9

Fisher, W.S., Fore, L.S., Hutchins, A., Quarles, R.L., Campbell, J.G., LoBue, C., Davis, W.S., 2008. Evaluation of stony coral indicators for coral reef management. Mar. Pollut. Bull. 56, 1737-45. doi:10.1016/j.marpolbul.2008.07.002

Fox, H.E., Caldwell, R.L., 2006. Recovery from blast fishing on coral reefs: a tale of two scales. Ecol. Appl. 16, 1631-1635. doi:10.1890/1051-0761(2006)016[1631:rfbfoc]2.0.co;2

Frazer, T.K., Jacoby, C.A., Edwards, M.A., Barry, S.C., Manfrino, C.M., 2012. Coping with the lionfish invasion: Can targeted removals yield beneficial effects? Rev. Fish. Sci. 20, 185-191. doi:10.1080/10641262.2012.700655

Garzón-Ferreira, J., Zea, S., Díaz, J.M., 2005. Incidence of partial mortality and other health indicators in hard-coral communities of four southwestern Caribbean atolls. Bull. Mar. Sci. 76, 105-122.

Gilbert, M., Rasmussen, J.B., Kramer, D.L., 2005. Estimating the density and biomass of moray eels (Muraenidae) using a modified visual census method for hole-dwelling reef fauna. Environ. Biol. Fishes 73, 415-426. doi:10.1007/s10641-005-2228-2

Gilmour, J.P., Smith, L.D., Heyward, A.J., Baird, A.H., Pratchett, M.S., 2013. Recovery of an Isolated Coral Reef System Following Severe Disturbance. Science 340, 69-71. doi:10.1126/science.1232310

Gladfelter, W.B., 1982. White-band disease in Acropora palmata: implications for the structure and growth of shallow reefs. Bull. Mar. Sci. 32, 639-643.

Glynn, P.W., 1997. Bioerosion and coral-reef growth: A dynamic balance, in: Birkeland, C. (Ed.), Life and Death of Coral Reefs. Chapman and Hall, New York, pp. 68-95. 
Goreau, T.J., Macfarlane, A.H., 1990. Reduced growth rate of Montastrea annularis following the 1987-1988 coral-bleaching event. Coral Reefs 8, 211-215. doi:10.1007/BF00265013

Graham, N.A.J., Dulvy, N.K., Jennings, S., Polunin, N.V.C., 2005. Size-spectra as indicators of the effects of fishing on coral reef fish assemblages. Coral Reefs 24, 118-124. doi:10.1007/s00338004-0466-y

Graham, N.A.J., Nash, K.L., Kool, J.T., 2011. Coral reef recovery dynamics in a changing world. Coral Reefs 30, 283-294. doi:10.1007/s00338-010-0717-z

Gratwicke, B., Speight, M.R., 2005. The relationship between fish species richness, abundance and habitat complexity in a range of shallow tropical marine habitats. J. Fish Biol. 66, 650-667. doi:10.1111/j.0022-1112.2005.00629.x

Green, S.J., Akins, J.L., Maljković, A., Côté, I.M., 2012. Invasive Lionfish Drive Atlantic Coral Reef Fish Declines. PLoS One 7, e32596. doi:10.1371/journal.pone.0032596

Haapkylä, J., Unsworth, R.K.F., Flavell, M., Bourne, D.G., Schaffelke, B., Willis, B.L., 2011. Seasonal Rainfall and Runoff Promote Coral Disease on an Inshore Reef. PLoS One 6, e16893. doi:10.1371/journal.pone.0016893

Harborne, A.R., Renaud, P.G., Tyler, E.H.M., Mumby, P.J., 2009. Reduced density of the herbivorous urchin Diadema antillarum inside a Caribbean marine reserve linked to increased predation pressure by fishes. Coral Reefs 28, 783-791. doi:10.1007/s00338-009-0516-6

Harrington, L., Fabricius, K., Eaglesham, G., Negri, A., 2005. Synergistic effects of diuron and sedimentation on photosynthesis and survival of crustose coralline algae. Mar. Pollut. Bull. 51, 415-427. doi:10.1016/j.marpolbul.2004.10.042

Harvell, D., Jordán-Dahlgren, E., Merkel, S., Rosenberg, E., Raymundo, L., Smith, G., Weil, E., Willis, B.L., 2007. Coral disease, environmental drivers, and the balance between coral and microbial associates. Oceanography 20, 172 - 195. doi:10.5670/oceanog.2007.91

Hatcher, B.G., 1984. A maritime accident provides evidence for alternate stable states in benthic communities on coral reefs. Coral Reefs 3, 199-204. doi:10.1007/BF00288255

Hawkins, J.P., Roberts, C.M., 2004. Effects of Artisanal Fishing on Caribbean Coral Reefs. Conserv. Biol. 18, 215-226. doi:10.1111/j.1523-1739.2004.00328.x

Hédouin, L., Berteaux-Lecellier, V., 2014. Traditional vs new approaches for assessing coral health: A global overview and the paradigm of French polynesia. J. Mar. Sci. Technol. 22, 25-35. doi:10.6119/JMST-013-0813-2

Heenan, A., Williams, I.D., 2013. Monitoring Herbivorous Fishes as Indicators of Coral Reef Resilience in American Samoa. PLoS One 8. doi:10.1371/journal.pone.0079604

Hill, J., Wilkinson, C.R., 2004. Methods for ecological monitoring of coral reefs: A resource for managers. Australian Institute of Marine Science, Townsville, Australia.

Hodgson, G., Hill, J., Kiene, W., Maun, L., Mihaly, J., Liebeler, J., Shuman, C., Torres, R., 2006. Reef Check Instruction Manual: A Guide to Reef Check Coral Reef Monitoring. Reef Check Foundation, Pacific Palisades, California, U.S.A.

Hoegh-Guldberg, O., 1999. Climate change, coral bleaching and the future of the world's coral reefs. Mar. Freshw. Res. 50, 839-866. doi:10.1071/MF99078

Hoey, A.S., Bellwood, D.R., 2009. Damselfish territories as a refuge for macroalgae on coral reefs. Coral Reefs 29, 107-118. doi:10.1007/s00338-009-0567-8

Holmes, K.E., 2000. Effects of eutrophication on bioeroding sponge communities with the 
description of new West Indian sponges, Cliona spp.(Porifera: Hadromerida: Clionidae). Invertebr. Biol. 119, 125-138. doi:10.1111/j.1744-7410.2000.tb00001.x

Houk, P., van Woesik, R., 2013. Progress and Perspectives on Question-Driven Coral-Reef Monitoring. Bioscience 63, 297-303. doi:10.1525/bio.2013.63.4.10

Hughes, T.P., Connell, J.H., 1999. Multiple stressors on coral reefs: A long-term perspective. Limnol. Oceanogr. 44, 932-940. doi:10.4319/lo.1999.44.3_part_2.0932

Hughes, T.P., Jackson, J.B.C., 1980. Do Corals Lie About Their Age? Some Demographic Consequences of Partial Mortality, Fission, and Fusion. Science 209, 713-715. doi:10.1126/science.209.4457.713

Hughes, T.P., Tanner, J.E., 2000. Recruitment failure, life histories, and long-term decline of Caribbean corals. Ecology 81, 2250-2263. doi:10.2307/177112

Igulu, M.M., Nagelkerken, I., Dorenbosch, M., Grol, M.G.G., Harborne, A.R., Kimirei, I.A., Mumby, P.J., Olds, A.D., Mgaya, Y.D., 2014. Mangrove Habitat Use by Juvenile Reef Fish: Meta-Analysis Reveals that Tidal Regime Matters More than Biogeographic Region. PLoS One 9, e114715. doi:10.1371/journal.pone.0114715

Jackson, J.B.C., Donovan, M.K., Cramer, K., Lam, V., 2014. Status and Trends of Caribbean Coral Reefs: 1970 - 2012. Global Coral Reef Monitoring Network, IUCN, Gland, Switzerland.

Jameson, S.C., Erdmann, M. V., Gibson, G.R., Potts, K.W., 1998. Development of biological criteria for coral reef ecosystem assessment. Atoll Res. Bull. 450, 1-102. doi:10.5479/si.00775630.450.1

Jameson, S.C., Erdmann, M., Karr, J.R., Potts, K.W., 2001. Charting a course toward diagnostic monitoring: A continuing review of coral reef attributes and a research strategy for creating coral reef indexes of biotic integrity. Bull. Mar. Sci. 69, 701-744.

Johnson, A.E., 2010. Reducing bycatch in coral reef trap fisheries: Escape gaps as a step towards sustainability. Mar. Ecol. Prog. Ser. 415, 201-209. doi:10.3354/meps08762

Jones, G.P., Almany, G.R., Russ, G.R., Sale, P.F., Steneck, R.S., Oppen, M.J.H., Willis, B.L., 2009. Larval retention and connectivity among populations of corals and reef fishes: history, advances and challenges. Coral Reefs 28, 307-325. doi:10.1007/s00338-009-0469-9

Jones, R.J., Bowyer, J., Hoegh-Guldberg, O., Blackall, L.L., 2004. Dynamics of a temperature-related coral disease outbreak. Mar. Ecol. Prog. Ser. 281, 63-77. doi:10.3354/meps281063

Karr, J.R., Chu, E.W., 1999. Restoring life in running waters: better biological monitoring. Island Press, Washington D.C.

Karr, K.A., Fujita, R., Halpern, B.S., Kappel, C. V, Crowder, L., Selkoe, K. a., Alcolado, P.M., Rader, D., 2015. Thresholds in Caribbean coral reefs: implications for ecosystem-based fishery management. J. Appl. Ecol. 52, 402-412. doi:10.1111/1365-2664.12388

Knowlton, N., 2001. The future of coral reefs. Proc. Natl. Acad. Sci. U. S. A. 98, 5419-25. doi:10.1073/pnas.091092998

Kramer, P., 2003. Synthesis of coral reef health indicators for the Western Atlantic: results of the AGRRA program (1997-2000). Atoll Res. Bull. 496, 1-58. doi:10.5479/si.00775630.496-3.1

Kramer, P.R., McField, M., Álvarez-Filip, L., Drysdale, I., Flores, M.R., Giro, A., Pott, R., 2015. 2015 Report Card for the Mesoamerican Reef. Healthy Reefs Initiative (www.healthyreefs.org)

Kubicek, A., Muhando, C., Reuter, H., 2012. Simulations of Long-Term Community Dynamics in Coral Reefs - How Perturbations Shape Trajectories. PLoS Comput. Biol. 8, e1002791. doi:10.1371/journal.pcbi.1002791 
Kuffner, I.B., Walters, L.J., Becerro, M.A., Paul, V.J., Ritson-Williams, R., Beach, K.S., 2006. Inhibition of coral recruitment by macroalgae and cyanobacteria. Mar. Ecol. Prog. Ser. 323, 107-117. doi:10.3354/meps323107

Kuguru, B.L., Mgaya, Y.D., Öhman, M.C., Wagner, G.M., 2004. The reef environment and competitive success in the Corallimorpharia. Mar. Biol. 145, 875-884. doi:10.1007/s00227-004-1376-9

Lang, J.C., Marks, K.W., Kramer, P.R., Ginsburg, R.N., 2010. AGRRA protocols version 5.4 [WWW Document]. URL http://www.agrra.org/method/AGRRA-V5.4_2010.pdf (accessed 10.22.15).

Legg, C.J., Nagy, L., 2006. Why most conservation monitoring is, but need not be, a waste of time. J. Environ. Manage. 78, 194-9. doi:10.1016/j.jenvman.2005.04.016

León, R. de, Vane, K., Bertuol, P., Chamberland, V.C., Simal, F., Imms, E., Vermeij, M.J.A., 2013. Effectiveness of lionfish removal efforts in the southern Caribbean. Endanger. Species Res. 22, 175-182. doi:10.3354/esr00542

Lesser, M.P., 2011. Coral Bleaching: Causes and Mechanisms, in: Dubinsky, Z., Stambler, N. (Eds.), Coral Reefs: An Ecosystem in Transition. Springer Netherlands, pp. 405-419. doi:10.1007/97894-007-0114-4_23

Lessios, H.A., 1988. Mass mortality of Diadema antillarum in the Caribbean: what have we learned? Annu. Rev. Ecol. Syst. 19, 371-393. doi:10.1146/annurev.es.19.110188.002103

Leujak, W., Ormond, R.F.G., 2007. Comparative accuracy and efficiency of six coral community survey methods. J. Exp. Mar. Bio. Ecol. 351, 168 - 187. doi:10.1016/j.jembe.2007.06.028

Lewis, J.B., 1997. Abundance, distribution and partial mortality of the massive coral Siderastrea siderea on degrading coral reefs at Barbados, West Indies. Mar. Pollut. Bull. 34, 622-627. doi:10.1016/S0025-326X(96)00184-1

Liu, G., Heron, S.F., Eakin, C.M., Muller-Karger, F.E., Vega-Rodriguez, M., Guild, L.S., De La Cour, J.L., Geiger, E.F., Skirving, W.J., Burgess, T.F.R., Strong, A.E., Harris, A., Maturi, E., Ignatov, A., Sapper, J., Li, J., Lynds, S., 2014. Reef-Scale Thermal Stress Monitoring of Coral Ecosystems: New 5-km Global Products from NOAA Coral Reef Watch. Remote Sens. 6, 11579-11606. doi:10.3390/rs61111579

Loh, T.-L., Pawlik, J.R., 2014. Chemical defenses and resource trade-offs structure sponge communities on Caribbean coral reefs. Proc. Natl. Acad. Sci. U. S. A. 111, 4151-4156. doi:10.1073/pnas.1321626111

Madin, J.S., 2005. Mechanical limitations of reef corals during hydrodynamic disturbances. Coral Reefs 24, 630-635. doi:10.1007/s00338-005-0042-0

Mah, A., Stearn, C., 1986. The effect of Hurricane Allen on the Bellairs fringing reef, Barbados. Coral Reefs 4, 169-176. doi:10.1007/bf00427938

Marshall, P., Schuttenberg, H., 2006. A reef manager's guide to coral bleaching. Great Barrier Reef Marine Park Authority, Townsville, Australia.

Marshall, P.A., Baird, A.H., 2000. Bleaching of corals on the Great Barrier Reef: differential susceptibilities among taxa. Coral Reefs 19, 155-163. doi:10.1007/s003380000086

Marshell, A., Mumby, P.J., 2015. The role of surgeonfish (Acanthuridae) in maintaining algal turf biomass on coral reefs. J. Exp. Mar. Bio. Ecol. 473, 152-160. doi:10.1016/j.jembe.2015.09.002

McClanahan, T.R., Ateweberhan, M., Graham, N.A.J., Wilson, S.K., Ruiz Sebastián, C., Guillaume, M.M.M., Bruggemann, J.H., 2007. Western Indian Ocean coral communities: bleaching responses and susceptibility to extinction. Mar. Ecol. Prog. Ser. 337, 1-13. 
doi:10.3354/meps337001

McClanahan, T.R., Cokos, B.A., Sala, E., 2002. Algal growth and species composition under experimental control of herbivory, phosphorus and coral abundance in Glovers Reef, Belize. Mar. Pollut. Bull. 44, 441-51. doi:10.1016/S0025-326X(02)00051-6

McClanahan, T.R., Donner, S.D., Maynard, J.A., MacNeil, M.A., Graham, N.A.J., Maina, J., Baker, A.C., Alemu I, J.B., Beger, M., Campbell, S.J., Darling, E.S., Eakin, C.M., Heron, S.F., Jupiter, S.D., Lundquist, C.J., McLeod, E., Mumby, P.J., Paddack, M.J., Selig, E.R., van Woesik, R., 2012. Prioritizing key resilience indicators to support coral reef management in a changing climate. PLoS One 7, e42884. doi:10.1371/journal.pone.0042884

McClanahan, T.R., Shafir, S.H., 1990. Causes and consequences of sea urchin abundance and diversity in Kenyan coral reef lagoons. Oecologia 83, 362-370. doi:10.1007/bf00317561

McClenachan, L., Kittinger, J.N., 2013. Multicentury trends and the sustainability of coral reef fisheries in Hawai'i and Florida. Fish Fish. 14, 239-255. doi:10.1111/j.1467-2979.2012.00465.x

McField, M., Kramer, P.R., 2007. Healthy Reefs for Health People: A guide to indicators of reef health and social well-being in the Mesoamerican reef region. With contributions by M. Gorrez and M. McPherson. (www.healthyreefs.org)

McField, M., Kramer, P.R., 2006. The Healthy Mesoamerican Reef Ecosystem Initiative: a conceptual framework for evaluating reef ecosystem health, in: Proceedings of the 10th International Coral Reef Symposium. Okinawa, Japan, pp. 1118-1123.

Miller, I.R., Logan, M., Johns, K.A., Jonker, M.J., Osborne, K., Sweatman, H.P.A., 2013. Determining background levels and defining outbreaks of crustose coralline algae disease on the Great Barrier Reef. Mar. Freshw. Res. doi:10.1071/MF12330

Moran, P.J., De'ath, G., 1992. Estimates of the abundance of the crown-of-throns starfish Acanthaster planci in outbreaking and non-outbreaking populations on reefs within the Great Barrier Reef. Mar. Biol. 113, 509-515. doi:10.1007/BF00349178

Morris, J.A.J., Akins, J.L., 2009. Feeding ecology of invasive lionfish (Pterois volitans) in the Bahamian archipelago. Environ. Biol. Fishes 86, 389-398. doi:10.1007/s10641-009-9538-8

Muhando, C.A., Kuguru, B.L., Wagner, G.M., Mbije, N.E., Ohman, M.C., 2002. Environmental effects on the distribution of corallimorpharians in Tanzania. Ambio 31, 558-561. doi:10.1579/00447447-31.7.558

Mumby, P.J., 1999. Bleaching and hurricane disturbances to populations of coral recruits in Belize. Mar. Ecol. Prog. Ser. 190, 27-35. doi:10.3354/meps190027

Mumby, P.J., Bejarano, S., Golbuu, Y., Steneck, R.S., Arnold, S.N., van Woesik, R., Friedlander, A.M., 2013. Empirical relationships among resilience indicators on Micronesian reefs. Coral Reefs 32 , 213-226. doi:10.1007/s00338-012-0966-0

Mumby, P.J., Dahlgren, C.P., Harborne, A.R., Kappel, C. V, Micheli, F., Brumbaugh, D.R., Holmes, K.E., Mendes, J.M., Broad, K., Sanchirico, J.N., Buch, K., Box, S., Stoffle, R.W., Gill, A.B., 2006. Fishing, Trophic Cascades, and the Process of Grazing on Coral Reefs. Science 311, 98-101. doi:10.1126/science.1121129

Mumby, P.J., Edwards, A.J., Arias-González, J.E., Lindeman, K.C., Blackwell, P.G., Gall, A., Gorczynska, M.I., Harborne, A.R., Pescod, C.L., Renken, H., Wabnitz, C.C.C., Llewellyn, G., 2004. Mangroves enhance the biomass of coral reef fish communities in the Caribbean. Nature 427, 533-536. doi:10.1029/2001JB001194

Mumby, P.J., Foster, N.L., Fahy, E.A.G., 2005. Patch dynamics of coral reef macroalgae under chronic 
and acute disturbance. Coral Reefs 24, 681-692. doi:10.1007/s00338-005-0058-5

Mumby, P.J., Hastings, A., Edwards, H.J., 2007. Thresholds and the resilience of Caribbean coral reefs. Nature 450, 98-101. doi:10.1038/nature06252

Mumby, P.J., Steneck, R.S., Adjeroud, M., Arnold, S.N., 2015. High resilience masks underlying sensitivity to algal phase shifts of Pacific coral reefs. Oikos. doi:10.1111/oik.02673

Mumby, P.J., Steneck, R.S., Edwards, A.J., Ferrari, R., Coleman, R., Harborne, A.R., Gibson, J.P., 2012. Fishing down a Caribbean food web relaxes trophic cascades. Mar. Ecol. Prog. Ser. 445, 13-24. doi:10.3354/meps09450

Mumby, P.J., van Woesik, R., 2014. Consequences of Ecological, Evolutionary and Biogeochemical Uncertainty for Coral Reef Responses to Climatic Stress. Curr. Biol. 24, R413-R423. doi:10.1016/j.cub.2014.04.029

Nagelkerken, I., Dorenbosch, M., Verberk, W., Cocheret de la Morinière, E., van der Velde, G., 2000. Day-night shifts of fishes between shallow-water biotopes of a Caribbean bay, with emphasis on the nocturnal feeding of Haemulidae and Lutjanidae. Mar. Ecol. Prog. Ser. 194, 55-64. doi:10.3354/meps194055

Newman, M.J.H., Paredes, G.A., Sala, E., Jackson, J.B.C., 2006. Structure of Caribbean coral reef communities across a large gradient of fish biomass. Ecol. Lett. 9, 1216-27. doi:10.1111/j.14610248.2006.00976.x

Norström, A. V, Nyström, M., Lokrantz, J., Folke, C., 2009. Alternative states on coral reefs: Beyond coral-macroalgal phase shifts. Mar. Ecol. Prog. Ser. 376, 295 - 306. doi:10.3354/meps07815

Nugues, M.M., Bak, R.P.M., 2006. Differential competitive abilities between Caribbean coral species and a brown alga: a year of experiments and a long-term perspective. Mar. Ecol. Prog. Ser. 315, 75-86. doi:10.3354/meps315075

Nugues, M.M., Roberts, C.M., 2003. Partial mortality in massive reef corals as an indicator of sediment stress on coral reefs. Mar. Pollut. Bull. 46, 314-23. doi:10.1016/S0025$326 \times(02) 00402-2$

Obura, D., Grimsditch, G., 2009. Resilience Assessment of coral reefs: Assessment protocol for coral reefs, focusing on coral bleaching and thermal stress. IUCN working group on Climate Change and Coral Reefs. IUCN, Gland, Switzerland.

Ohlhorst, S.L., Liddell, W.D., Taylor, R.J., Taylor, M.J., 1988. Evaluation of reef census techniques, in: Proceedings of the 6th International Coral Reef Symposium, Townsville, Australia, pp. 319-324.

Ortiz, J.C., Bozec, Y.-M., Wolff, N.H., Doropoulos, C., Mumby, P.J., 2014a. Global disparity in the ecological benefits of reducing carbon emissions for coral reefs. Nat. Clim. Chang. 4, 10901094. doi:10.1038/nclimate2439

Ortiz, J.C., González-Rivero, M., Mumby, P.J., 2014b. An Ecosystem-Level Perspective on the Host and Symbiont Traits Needed to Mitigate Climate Change Impacts on Caribbean Coral Reefs. Ecosystems 17, 1-13. doi:10.1007/s10021-013-9702-z

Osborne, K., Dolman, A.M., Burgess, S.C., Johns, K.A., 2011. Disturbance and the Dynamics of Coral Cover on the Great Barrier Reef (1995-2009). PLoS One 6, e17516. doi:10.1371/journal.pone.0017516

Pawlik, J.R., Loh, T.-L., McMurray, S.E., Finelli, C.M., 2013. Sponge communities on Caribbean coral reefs are structured by factors that are top-down, not bottom-up. PLoS One 8, e62573. doi:10.1371/journal.pone.0062573 
Pratchett, M.S., Caballes, C., Rivera--Posada, J., Sweatman, H., 2014. Limits to Understanding and Managing Outbreaks of Crown-of-Thorns Starfish (Acanthaster spp.). Oceanogr. Mar. Biol. An Annu. Rev. 52, 133-200. doi:10.1201/b17143-4

Quéré, G., Steneck, R.S., Nugues, M.M., 2015. Spatiotemporal and species-specific patterns of diseases affecting crustose coralline algae in Curaçao. Coral Reefs 34, 259-273. doi:10.1007/s00338-014-1225-3

Randall, C.J., van Woesik, R., 2015. Contemporary white-band disease in Caribbean corals driven by climate change. Nat. Clim. Chang. doi:10.1038/nclimate2530

Rasher, D.B., Hay, M.E., 2010. Chemically rich seaweeds poison corals when not controlled by herbivores. Proc. Natl. Acad. Sci. U. S. A. 107, 9683-8. doi:10.1073/pnas.0912095107

Rasser, M., Riegl, B., 2002. Holocene coral reef rubble and its binding agents. Coral Reefs 21, 57-72. doi:10.1007/s00338-001-0206-5

Raymundo, L., Couch, C.S., Harvell, C.D., 2008. Coral disease handbook: guidelines for assessment, monitoring and management. Coral Reef Targeted Research \& Capacity Building for Management Programme: St Lucia, Australia.

Renken, H., Mumby, P.J., 2009. Modelling the dynamics of coral reef macroalgae using a Bayesian belief network approach. Ecol. Modell. 220, 1305-1314. doi:10.1016/j.ecolmodel.2009.02.022

Risk, M.J., 1999. Paradise lost: how marine science failed the world's coral reefs. Mar. Freshw. Res. doi:10.1071/MF99067

Risk, M.J., 1972. Fish diversity on a coral reef in the Virgin Islands. Atoll Res. Bull. 153, 1-4. doi:10.5479/si.00775630.153.1

Risk, M.J., Edinger, E., 2011. Impacts of Sediment on Coral Reefs, in: Hopley, D. (Ed.), Encyclopedia of Modern Coral Reefs SE - 25, Encyclopedia of Earth Sciences Series. Springer Netherlands, pp. 575-586. doi:10.1007/978-90-481-2639-2_25

Ritson-Williams, R., Arnold, S.N., Fogarty, N.D., Steneck, R.S., Vermeij, M.J.A., Paul, V.J., 2009. New Perspectives on Ecological Mechanisms Affecting Coral Recruitment on Reefs. Smithson. Contrib. to Mar. Sci. 38, 437-457. doi:10.5479/si.01960768.38.437

Roff, G., Mumby, P.J., 2012. Global disparity in the resilience of coral reefs. Trends Ecol. Evol. 27, 404-13. doi:10.1016/j.tree.2012.04.007

Rogers, C.S., 1990. Responses of coral reefs and reef organisms to sedimentation. Mar. Ecol. Prog. Ser. 62, 185-202. doi:10.3354/meps062185

Rose, C.S., Risk, M.J., 1985. Increase in Cliona delitrix infestation of Montastrea cavernosa heads on an organically polluted portion of the Grand Cayman fringing reef. Mar. Ecol. 6, 345-363. doi:10.1111/j.1439-0485.1985.tb00142.x

Rotjan, R., Lewis, S., 2008. Impact of coral predators on tropical reefs. Mar. Ecol. Prog. Ser. 367, 7391. doi:10.3354/meps07531

Ruiz-Moreno, D., Willis, B.L., Page, A.C., Weil, E., Cróquer, A., Vargas-Angel, B., Jordan-Garza, A.G., Jordán-Dahlgren, E., Raymundo, L., Harvell, C.D., 2012. Global coral disease prevalence associated with sea temperature anomalies and local factors. Dis. Aquat. Organ. 100, 249-61. doi:10.3354/dao02488

Russ, G.R., 2003. Grazer biomass correlates more strongly with production than with biomass of algal turfs on a coral reef. Coral Reefs 22, 63-67. doi:10.1007/s00338-003-0286-5

Russ, G.R., 1984. Distribution and abundance of herbivorous grazing fishes in the central Great 
Barrier reef. 1. Levels of variability across the entire continental-shelf. Mar. Ecol. Prog. Ser. 20, 23-34. doi:10.3354/meps020023

Russ, G.R., McCook, L.J., 1999. Potential effects of a cyclone on benthic algal production and yield to grazers on coral reefs across the central Great Barrier Reef. J. Exp. Mar. Bio. Ecol. 235, 237-254. doi:10.1016/S0022-0981(98)00180-4

Schönberg, C.H.L., Ortiz, J.C., 2009. Is sponge bioerosion increasing?, in: Proceedings of the 11th International Coral Reef Symposium 2008, Ft. Lauderdale, Florida, pp. 527-530.

Scoffin, T.P., 1993. The geological effects of hurricanes on coral reefs and the interpretation of storm deposits. Coral Reefs 12, 203-221. doi:10.1007/BF00334480

Smith, S. V, Kimmerer, W.J., Laws, E.A., Brock, R.E., Walsh, T.E., 1981. Kaneohe Bay sewage diversion experiment: perspectives on ecosystem response to nutritional perturbation. Pacific Sci. 35, 279-395. doi: 10125/616

Steneck, R.S., 1997. Crustose corallines, other algal functional groups, herbivores and sediments: complex interactions along reef productivity gradients, in: Lessios, H.A., Macintyre, I.G. (Eds.), Proceedings of the 8th International Coral Reef Symposium. Smithsonian Tropical Research Institute, Panama, pp. 695-700.

Steneck, R.S., Arnold, S.N., León, R. de, Rasher, D.B., 2016. Status and trends of Bonaire's Coral Reefs in 2015: Slow but steady signs of resilience. Unpublished report to STINAPA Bonaire: University of Maine Darling Marine Center.

Steneck, R.S., McClanahan, T.R., Arnold, S.N., Brown, J.B., 2005. A Report on the Status of the Coral Reefs of Bonaire in 2005 with Advice on a Monitoring Program. Unpublished report to STINAPA Bonaire: University of Maine Darling Marine Center.

Stoner, A.W., Davis, M.H., Booker, C.J., 2012. Negative consequences of allee effect are compounded by fishing pressure: comparison of queen conch reproduction in fishing grounds and a marine protected area. Bull. Mar. Sci. 88, 89 - 104. doi:10.5343/bms.2011.1044

Sweatman, H., Cheal, A., Coleman, G., Emslie, M., Johns, K., Jonker, M., Miller, I., Osborne, K., 2008. Long-term monitoring of the Great Barrier Reef. Status Report Number 8. Australian Institute of Marine Science,Townsville, Australia.

Tanner, J.E., 1995. Competition between scleractinian corals and macroalgae: an experimental investigation of coral growth, survival and reproduction. J. Exp. Mar. Bio. Ecol. 190, 151-168. doi:10.1016/0022-0981(95)00027-0

Thurber, R.L.V., Burkepile, D.E., Fuchs, C., Shantz, A.A., McMinds, R., Zaneveld, J.R., 2013. Chronic nutrient enrichment increases prevalence and severity of coral disease and bleaching. Glob. Chang. Biol. 20, 544-554. doi:10.1111/gcb.12450

Tissot, B.N., Hallacher, L.E., 2003. Effects of Aquarium Collectors on Coral Reef Fishes in Kona, Hawaii. Conserv. Biol. 17, 1759-1768. doi:10.1111/j.1523-1739.2003.00379.x

Torres, J.L., 2001. Impacts of sedimentation on the growth rates of Montastraea annularis in southwest Puerto Rico. Bull. Mar. Sci. 69, 631-637.

van Woesik, R., Gilner, J., Hooten, A.J., 2009. Standard operating procedures for repeated measures of process and state variables of coral reef environments. Coral Reef Targeted Research \& Capacity Building for Management Programme: St Lucia, Australia.

Vermeij, M.J.A., Sandin, S.A., 2008. Density-dependent settlement and mortality structure the earliest life phases of a coral population. Ecology 89, 1994-2004. doi:10.1890/07-1296.1 
Vermeij, M.J.A., van Moorselaar, I., Engelhard, S., Hörnlein, C., Vonk, S.M., Visser, P.M., 2010. The effects of nutrient enrichment and herbivore abundance on the ability of turf algae to overgrow coral in the Caribbean. PLoS One 5, e14312. doi:10.1371/journal.pone.0014312

Wabnitz, C.C.C., Taylor, M., Green, E., Razak, T., 2003. From Ocean to Aquarium: The Global Trade in Marine Ornamental Species. UNEP-WCMC, Cambridge, UK.

Ward-Paige, C.A., Risk, M.J., Sherwood, O.A., Jaap, W.C., 2005. Clionid sponge surveys on the Florida Reef Tract suggest land-based nutrient inputs. Mar. Pollut. Bull. 51, 570-579. doi:10.1016/j.marpolbul.2005.04.006

Webster, N.S., 2007. Sponge disease: a global threat? Environ. Microbiol. 9, 1363-1375. doi:10.1111/j.1462-2920.2007.01303.x

Weil, E., Rogers, C.S., 2011. Coral reef diseases in the Atlantic-Caribbean, in: Dubinsky, Z., Stambler, N. (Eds.), Coral Reefs: An Ecosystem in Transition. Springer, pp. 465-491. doi:10.1007/978-94007-0114-4_27

Wilkinson, C.R., 2008. Status of coral reefs of the world: 2008. Global Coral Reef Monitoring Network and Reef and Rainforest Research Centre, Townsville, Australia.

Williams, I.D., Polunin, N.V.C., 2001. Large-scale associations between macroalgal cover and grazer biomass on mid-depth reefs in the Caribbean. Coral Reefs 19, 358-366. doi:10.1007/s003380000121

Williams, I.D., Polunin, N.V.C., Hendrick, V.J., 2001. Limits to grazing by herbivorous fishes and the impact of low coral cover on macroalgal abundance on a coral reef in Belize. Mar. Ecol. Prog. Ser. 222, 187-196. doi:10.3354/meps222187

Wilson, S.K., Fisher, R., Pratchett, M.S., Graham, N.A.J., Dulvy, N.K., Turner, R.A., Cakacaka, A., Polunin, N.V.C., Rushton, S.P., 2008. Exploitation and habitat degradation as agents of change within coral reef fish communities. Glob. Chang. Biol. 14, 2796-2809. doi:10.1111/j.13652486.2008.01696.x

Wolff, N.H., Donner, S.D., Cao, L., Iglesias-Prieto, R., Sale, P.F., Mumby, P.J., 2015. Global inequities between polluters and the polluted: climate change impacts on coral reefs. Glob. Chang. Biol. 21, 3982-3994. doi:10.1111/gcb.13015

Woodley, J., Chornesky, E., Clifford, P., Jackson, J.B.C., Kaufman, L.S., Knowlton, N., Lang, J.C., Pearson, M.P., Porter, J.W., Rooney, M.C., Rylaarsdam, K.W., Tunnicliffe, V.J., Wahle, C.M., Wulff, J.L., Curtis, A.S.G., Dallmeyer, M.D., Jupp, B.P., Koehl, M.A.R., Neigel, J., Sides, E.M., 1981. Hurricane Allen's impact on Jamaican coral reefs. Science 214, $749-755$. doi:10.1126/science.214.4522.749

Wynne, S.P., Côté, I.M., 2007. Effects of habitat quality and fishing on Caribbean spotted spiny lobster populations. J. Appl. Ecol. 44, 488-494. doi:10.1111/j.1365-2664.2007.01312.x 